\title{
Inkjet Printed, Self Powered, Wireless Sensors for Environmental, Gas, and Authentication-Based Sensing
}

\author{
Rushi Vyas, Student Member, IEEE, Vasileios Lakafosis, Student Member, IEEE, Hoseon Lee, \\ George Shaker, Member, IEEE, Li Yang, Student Member, IEEE, Giulia Orecchini, Anya Traille, \\ Manos M. Tentzeris, Fellow, IEEE, and Luca Roselli, Senior Member, IEEE
}

(Invited Paper)

\begin{abstract}
In this paper, inkjet-printed flexible sensors fabricated on paper substrates are introduced as a system-level solution for ultra-low-cost mass production of UHF Radio Frequency Identification (RFID) Tags and wireless sensor nodes in a "green" approach that could be easily extended to other microwave and wireless applications. The authors briefly touch up the state-of-the-art area of fully integrated wireless sensor modules on paper and show several active and power scavenging platforms to power on wireless sensors that could potentially set the foundation for the truly convergent wireless sensor ad hoc networks of the future.

Plus, the authors address the integration of carbon-nanotubes on paper substrates for the realization of ultra sensitive (parts per billion) gas sensors and present benchmarking results for various scavenging approaches involving solar and charge transfer-based mechanisms. Various challenges of packaging, passives, antennas, sensors and power sources integration are investigated in terms of ruggedness, reliability and flexibility performance for space, automotive, "smart-skin" and wearable applications.
\end{abstract}

Index Terms-Antennas, battery-less, carbon nanotube, "green" electronics, inkjet-printed electronics, low-power, passives, radio frequency (RF), radio frequency identification (RFID), sensor, solar, system-onpackage (SOP), system-on-paper, wireless.

\section{INTRODUCTION}

$\mathbf{T}$ HE electronics industry largely relies on traditional PCB fabrication methods, such as photo lithography, mechanical milling, or screen printing techniques. The vast majority

Manuscript received July 16, 2011; revised August 12, 2011; accepted August 13, 2011. Date of publication September 01, 2011; date of current version October 28, 2011. This work was supported in part by the National Science Foundation (NSF), the New Energy and Industrial Technology Development Organization (NEDO), and the Interconnect Focus Center (IFC). The associate editor coordinating the review of this paper and approving it for publication was Prof. Krikor Ozanyan.

R. Vyas, V. Lakafosis, H. Lee, A. Traille, and M. M. Tentzeris are with the Georgia Institute of Technology, Atlanta, GA 30332 USA (e-mail: Rushi.Vyas@gatech.edu; vasileios@gatech.edu; hoseon.lee@gmail.com; atraille@ece.gatech.edu; etentze@ece.gatech.edu).

G. Shaker is with the University of Waterloo, ON N2L 3G1, Canada (e-mail: gshaker@uwaterloo.ca).

L. Yang is with the Georgia Institute of Technology, Atlanta, GA 30332 USA. He is also with the Texas Instruments, Dallas, TX 75243 USA (e-mail: liyanggt@gmail.com).

G. Orecchini and L. Roselli are with the University of Perugia, 06123 Perugia, Italy (e-mail: giulia.orecchini@gmail.com).

Color versions of one or more of the figures in this paper are available online at http://ieeexplore.ieee.org.

Digital Object Identifier 10.1109/JSEN.2011.2166996 of circuits fabricated on PCB use FR-4, while screen printing is often used in fabricating circuits on PET commonly found in radio frequency identification (RFID) in-lays and tags. Both FR-4, which is a ceramic-based material and polyethylene terepthalate (PET) are nonbiodegradable substances that take decades to break down in landfills largely contributing to the bulk of the electronic waste generated annually. In this paper, we present a new "system-on-paper" approach that utilizes a very efficient and low-cost inkjet printing method to print "green" conformal electronic circuits on paper. Paper is highly biodegradable requiring a few months to break down organically in landfills. Due to their affinity to water, organic materials do tend to be more lossy at higher frequencies; nevertheless, as the authors have shown in [1], by using the correct paper and printing process, acceptable radio frequency (RF) attributes can be achieved on paper-based RF circuit designs. We demonstrate the successful use of the proposed inkjet printing technique on paper/polymer-based substrates for a very diverse range of applications integrating active and passive circuitry spanning a wide range of frequencies from LF/DC to ultra-high frequency (UHF) and super high frequency (SHF). On the passive side, we present: An artificial magnetic surface that could be effectively used as a frequency-selective shielding structure as a well as an enabling structure for ultra-thin RF modules; a couple of novel RFID-based "zero-power" sensors that utilize Carbon nanotubes and backscattering principles to eliminate the need of an RFIC at UHF frequencies around $900 \mathrm{MHz}$ bridging RF and Nanotechnology on paper substrates; a new method to couple inkjet printed patterns on paper along with an RF/EM decoding technique to realize RF hardware-authentication devices operating around 5-6 GHz. On the integrated side, we present a battery operated RFID-based wireless temperature sensor module operating at $900 \mathrm{MHz}$; and a new topology of realizing paper-based "battery-less" wireless modules using smaller solar cell arrays in order to achieve RFID functionality, which we refer to as semi active tag.

\section{InKJet PRinting Versus Wet Etching AND MILLING: TRADEOFFS}

Traditional wet etching techniques that form the backbone of the conventional PCB fabrication process involve complete metallization of substrate laminates and subsequent selective etching of portions of the metal layer on top of the substrate 


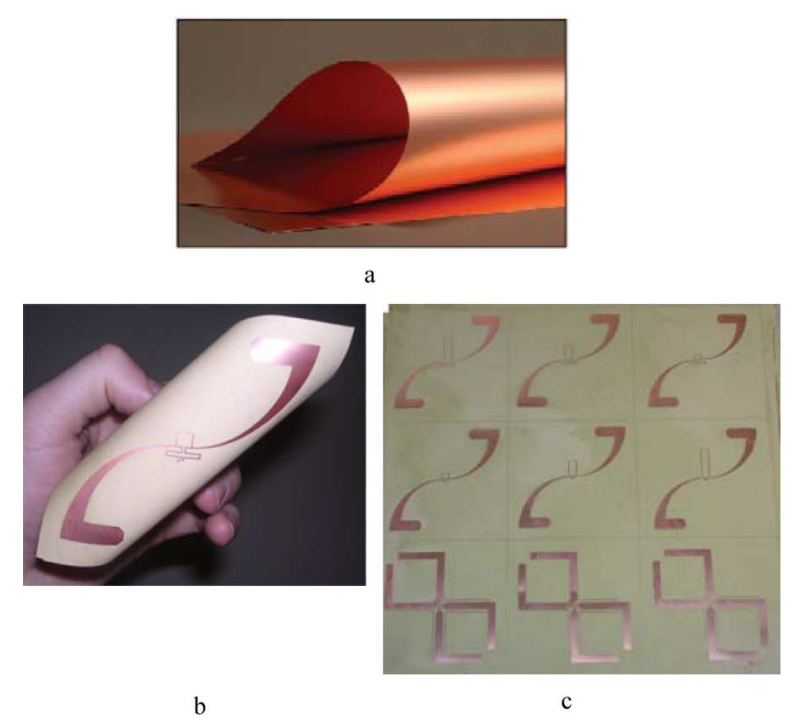

Fig. 1. (a) Unetched flexible metalized liquid crystal polymer laminates [2] (b) Etched RFID antennas on liquid crystal polymer using wet etching. (c) Reel to reel fabrication of RFID antenna on liquid crystal polymer.

using etchants (Fig. 1). Common wet etchants are solvents containing deionized water mixed with highly corrosive acids, such as nitric acid, sulfuric acid, and hydrofluoric acid. In addition to being hazardous to their handlers in cleanrooms, the washed out etchants combined with the discarded metal particles constitute a significant amount of waste in the circuit fabrication process, that require special treatment predisposal.

Milling machines are also used to realize circuits on metal laminated substrates. They operate like plotting machines with milling bits that selectively rub out unwanted metal off PCB boards. While they do have the advantage of not generating any etchant waste, they do generate metal files that are milled out of the board. A drawback of these machines is that they use a combination of servo motors not just to move the plotting arms but also push down and rotate the milling bits as they rub out the metal, which makes them very power hungry. In addition, their minimum pitch is $100 \mu \mathrm{m}$ in contrast to below $50 \mu \mathrm{m}$ for inkjet printing and wet etching methods making printing relatively more imprecise for frequencies beyond UHF. Laser milling machines have precisions comparable to inkjet printing $(\sim 50 \mu \mathrm{m})$ [3] but require high amounts of power of the order of $\mathrm{kW}$ and are unsuitable for metal cutting on thinner and flexible substrates such as paper and PET.

Inkjet printing of conductive material over a substrate offers substantial benefits over the previous methods in terms of reduced waste and ease of functionality. It typically uses an electrostatic potential $(5-12 \mathrm{~V})$ [4] difference between a charged electrode attached to the ink cartridge and the base plate on which the substrate is mounted. This results in the charged ink particles to ooze out of the nozzle and deposit on the substrate. The ink once printed is sintered on the substrate solidifies and forms the conductive layer on the substrate. The ink cartridge is mounted on a mechanical arm that is servo controlled to move in the $x$-direction and the base plate on which the substrate is mounted moves in the $y$-direction. This allows inkjet printing process to selectively metalize the substrate causing no material waste, as shown in Fig. 2.

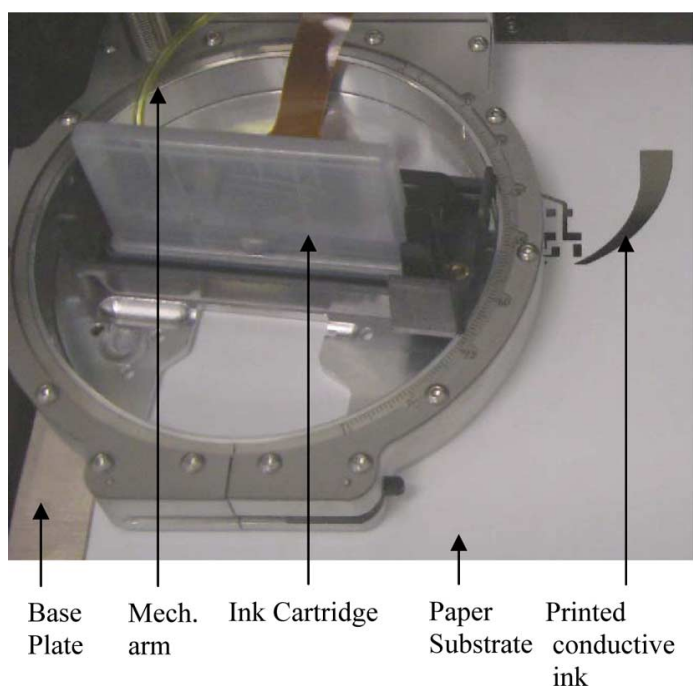

Fig. 2. Inkjet printed conductor on paper-based substrate.

The frugal and "green" nature of inkjet printing for fabricating substrates on low-cost organic flexible substrates such as paper make it ideal for large scale reel-to-reel processing with very high repeatability. This process is particularly ideal for large-area circuits which do not contain large metal areas, such as wireless sensors and 3D RF communication and control modules.

\section{MATERIAL DEPOSITION With INKJET PRINTING: VERSATILITY}

In addition to creating zero material waste through its selective ink spraying ability, inkjet printing also offers a unique versatility with regards to material deposition. Conventional cleanroom processes use different equipment for variable material deposition on substrates, such as different DC sputtering machines to shoot different metals in plasma form onto substrates typically at very low pressure. Nonmetallic materials are typically deposited using RF sputterers or ion deposition, which have to be etched out as a second step to get the desired material pattern on the substrate. Unlike bombarding substrates with plasma or ion vapor, inkjet printing uses electrostatic potential to deposit charged ink solvents in inkjet cartridges onto a substrate. The print head spays out controlled amounts of ink on it through multiple microscopic nozzles $254 \mu \mathrm{m}$ apart each about $21.5 \mu \mathrm{m}$ in diameter (Fig. 3) [4]. The print head temperature can be user-controlled (up to $70 \mathrm{C}$ ) in order to lower the viscosity of the ink solvents to between 10-12 cPs. The series of nozzles also have a filter to leave out large particles from the deposited ink size around $0.2 \mu \mathrm{m}$. Solvents containing the desired deposits can be easily formulated by combining material to be deposited with agents they are soluble in. The versatility of inkjet printing with regards to material deposition make it ideal for embedding sensing elements as well as functionalized nanostructures (such as Carbon Nanotubes-CNTs) onto circuit platforms for wireless or near- filed sensor telemetry constituting an extremely low-cost alternative to conventional material deposition technologies for the rapid and large-scale prototyping of low-power/zero-power RFID-enabled wireless sensors. 


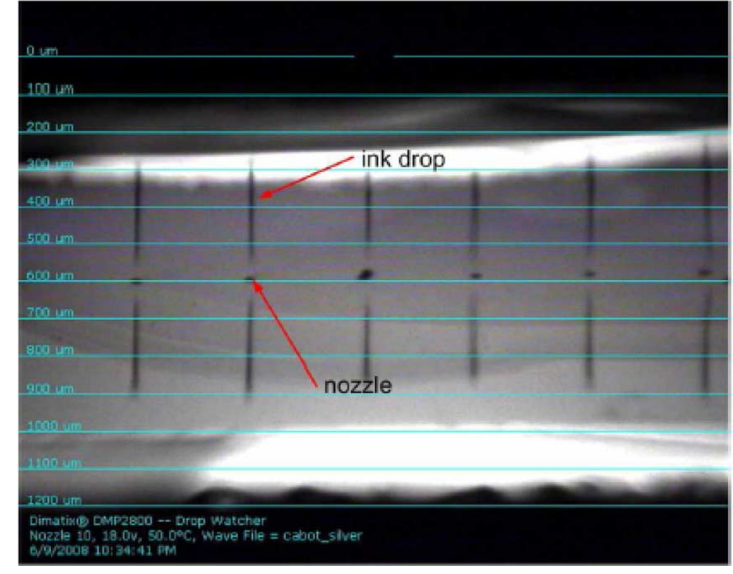

Fig. 3. Ink printed out of series of nozzles on print head.

\section{INKJET PRINTING: ACCURACY AND FORM}

IN addition to cutting down on waste and providing an all-in-one alternative to cleanroom deposition technologies, inkjet-printing attributes with regards to accuracy and form make it a very good contender for RF circuits. While the feasibility of inkjet printing for applications at UHF frequencies has been well covered under [1] and [6], its usability for application at even higher frequencies have not been investigated thoroughly. Operating at even higher frequencies in the SHF range $(3-30 \mathrm{GHz})$ require metallization that have relatively higher conductivities, low surface roughness, and with even smaller dimensions. The ink is sprayed in the $x$-direction, while the base plate with the substrate on moves in the $y$-direction to print an area. Due to this the resistance of the sprayed ink could be different in the $x$-direction from the $y$-direction. Ensuring uniform conductivity over the printed structure especially at microwave frequencies requires that the sprayed ink once treated has uniform distribution in both the $x$ and $y$ directions. Getting such a uniform surface profile with inkjet printing requires choosing the correct ink, the correct spray volume, printer settings, and ink treatment after it has been printed on the substrate. Using a print head capable of spraying out ink at a higher volume ensures a better overlap between adjacently sprayed conductive ink particles as they blot a little once being deposited on the substrate surface. This helps increase conductivity in both $x$ and $y$ directions. Fig. 4(a) and (b) shows the surface profile of an RFID antenna printed using cartridges spraying out ink droplets with volumes of 1 and $10 \mathrm{pL}$, respectively. In the former case, the striations along the antenna in the $y$-direction lead to an increased resistance in that direction compared to the latter case where a higher print volume ensure an overlap between each successively printed layer in the $y$-direction. The resulting range of the RFID in the latter case was increased almost by a factor of 100 , as shown in [6] and [7]. The resistance of the printed structure can also be further reduced by using a higher print resolution setting on the printer. RF circuits printed and tested using this technique has shown a printing resolution of 1000 dpi suitable for frequencies in the UHF range.

After printing, the deposited ink also needs to be annealed under the right conditions to ensure adjacent silver nanoparticles in the sprayed ink to gel with it neighbors allowing for a
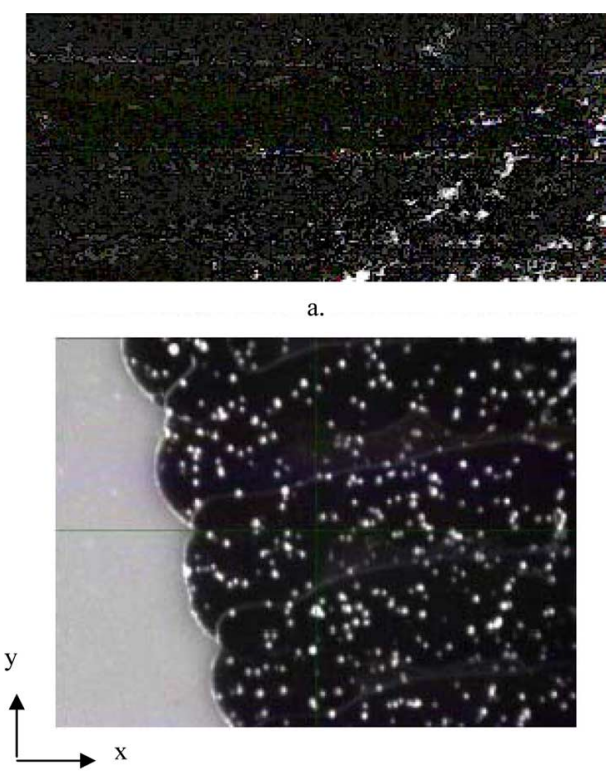

b.

Fig. 4. (a) Printed conductive ink layer with a volume of 1 pL. (b) Printed conductive ink layer with a volume of $10 \mathrm{pL}$.

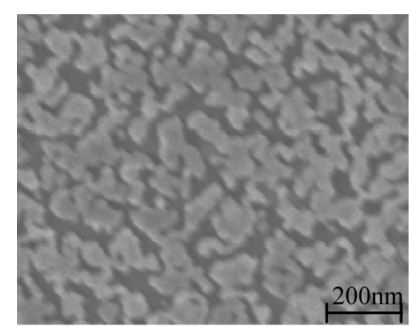

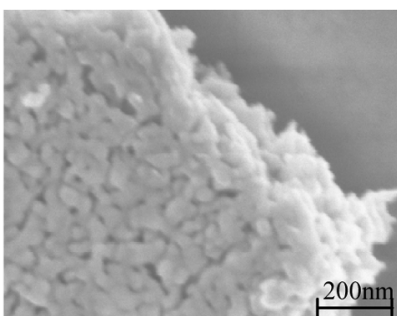

Fig. 5. SEM images of a layer of printed silver nanoparticle ink, after a $15 \mathrm{~min}$ cure at: (a) $100^{\circ} \mathrm{C}$ and (b) $150{ }^{\circ} \mathrm{C}$.

more uniform path for electron flow especially at microwave frequencies. The preannealing and postannealing effects on the conductive silver nanoparticles in the printed ink, as observed under an SEM, are shown in Fig. 5 [1]. Structures with very good conductivity and fine pitches suitable for frequencies well above UHF can be very accurately fabricated using the inkjet printing process, as shown by the close-up of a printed transmission line in Fig. 6.

\section{Discrete Circuit COMPONENT INTEGRATION ON PAPER}

With a dielectric permittivity of 3.1 , that is very stable well into the UHF range (variation between $3.05-3.15$ up to $15 \mathrm{GHz}$ ), paper offers a bio-friendly/ecofriendly alternative to ceramicbased substrates such as FR-4 (dielectric permittivity $=3.2$ ), which are also operable up to the UHF range, as well as to current wasteful PCB and PET circuit fabrication techniques. The low-temperature tolerance of the paper $\left(100{ }^{\circ} \mathrm{C}-110^{\circ} \mathrm{C}\right)$, however, does make soldering discrete components on it unfeasible. Nevertheless, highly conductive silver epoxy does provide a very reliable solution given its relatively low temperature curing requirement. At room temperatures, silver epoxies tend to be free flowing but harden and attach themselves to the printed silver pads when cured at higher temperatures between 


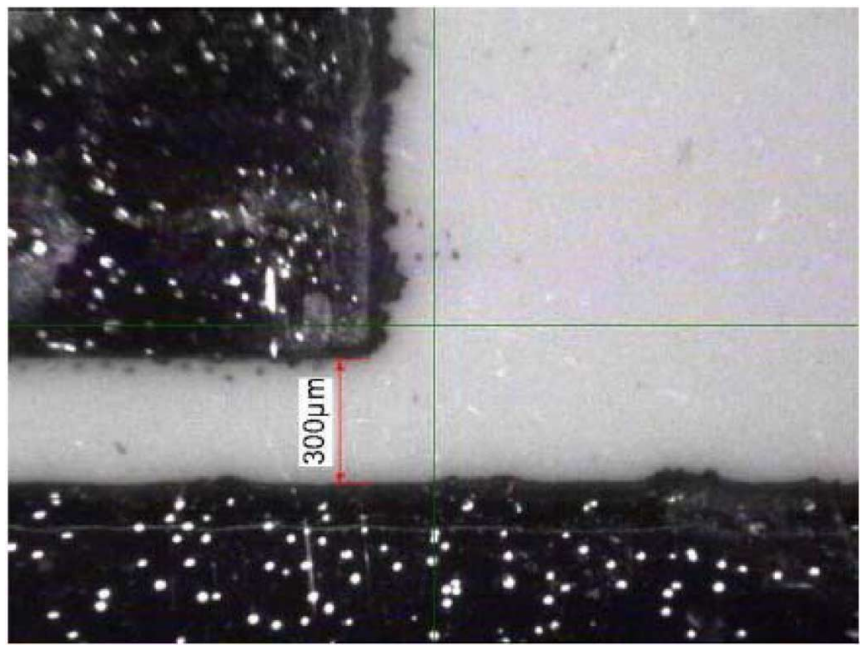

Fig. 6. Fiducial image of a CPW line inkjet printed for microwave frequencies.

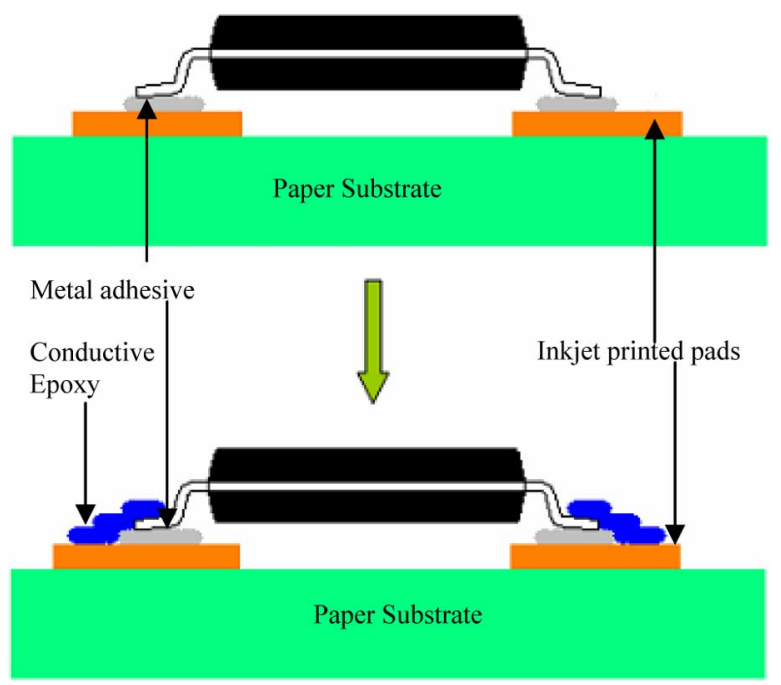

Fig. 7. IC component mounting process on inkjet printed silver pads.

$60{ }^{\circ} \mathrm{C}$ and $100{ }^{\circ} \mathrm{C}$ for a certain period of time. In detail, discrete IC components can be mounted on the inkjet printed circuit layout using a two-step process, as shown in Fig. 7. The first step involves holding the components in place on the printed silver pads with the use of a very small amount of adhesive between the component terminals and printed silver pads. Once in place, conductive silver epoxy can be afterwards applied on the terminals of the components to establish a conductive path between the terminals and printed silver pads [5]. A close-up of the free flowing silver-based epoxy manufactured by epo-tek on a printed coplanar waveguide pads (CPW) is shown in Fig. 8. The viscosity of the epo-tek epoxy (50\%-50\% mixture of resin and hardener) was low enough for it to percolate through via holes drilled through paper.

\section{ARTificial Magnetic SURFACES FOR UHF APPLICATION}

Artificial magnetic conductors, also known as high impedance surfaces, are structures that are capable of reflecting the incident electromagnetic waves at zero reflection

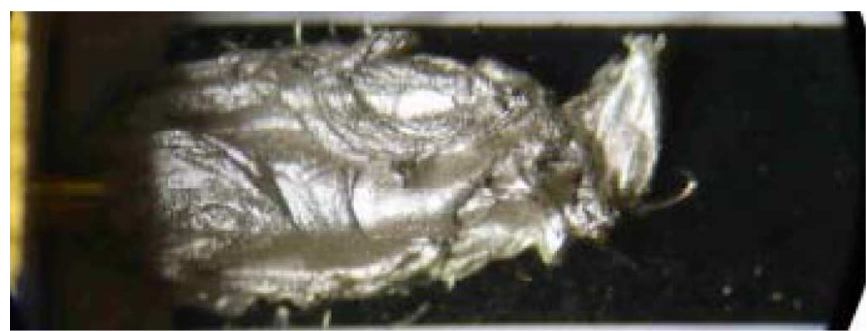

Fig. 8. Silver epoxy on printed silver pad.

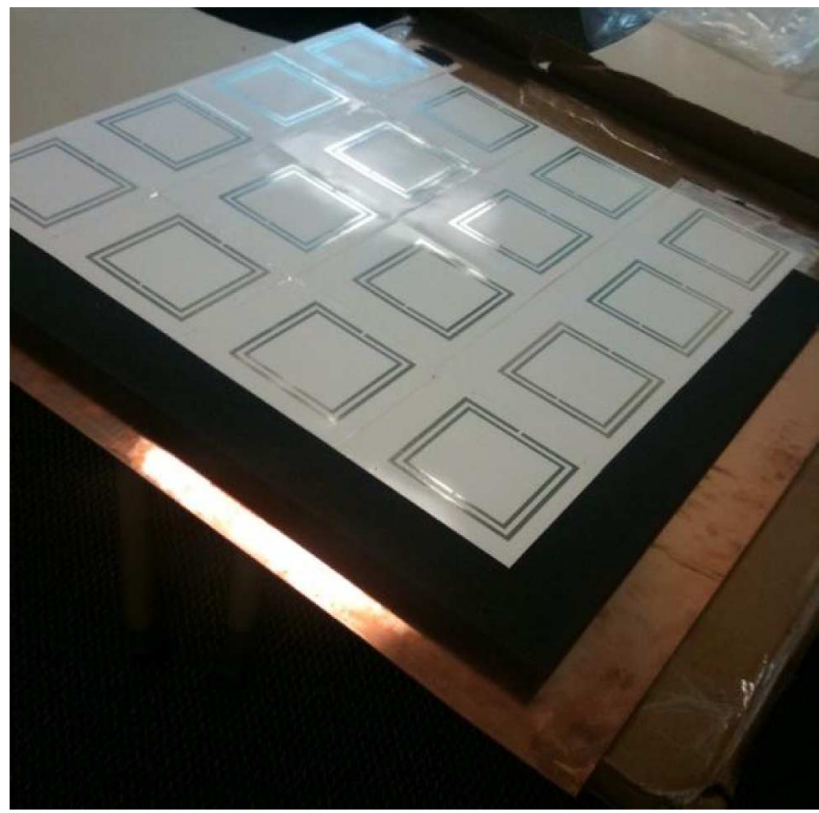

Fig. 9. Printed array of SRRs forming artificial magnetic surface.

phases, unlike metals or any electric conductor that enforces phase reversal for reflected waves. These structures can provide frequency-selective shielding solutions for multilayer RF modules as well as performance-enhancing structures for miniaturized/ultra thin antennas. The surface consists of an array of multiple split ring resonators (SRRs) to enable its multiband operability. Such an engineered surface has the potential in realizing low-profile ultra-thin antennas. The featured surface was designed utilizing the actual inkjet-printed silver ink conductivity $1 * 10^{7} \mathrm{~S} * \mathrm{~m}^{-1}$. As in [1], the dielectric constant (3.1) and loss tangent (0.06) of a 254 micron sheet of paper were used. In this work, the Dimatix DMP-2800 printer was used to print the SRRs. The unit cell has a size of $150 \mathrm{~mm} \times 50 \mathrm{~mm}$. The resulting ink thickness measured using Wyko profilometer, was around $3 \mu \mathrm{m}$ with a consistent measured conductivity of in the range $9 * 10^{6} \mathrm{~S} * \mathrm{~m}^{-1}-1 \cdot 1 * 10^{7} \mathrm{~S} * \mathrm{~m}^{-1}$ [8]. The SRR structure on paper was backed by a $25 \mathrm{~mm}$ thick foam layer on top of a metallic ground plane. The final prototype developed can be seen in Fig. 9 and the phase reflection of the electromagnetic wave normally incident on the surface is shown in Fig. 10 [8]. It is obvious that such inkjet-printed systems-on-paper could set the foundation for conformal ultra thin distributed antennas and packages that could be wearable or vehicle-mountable. 


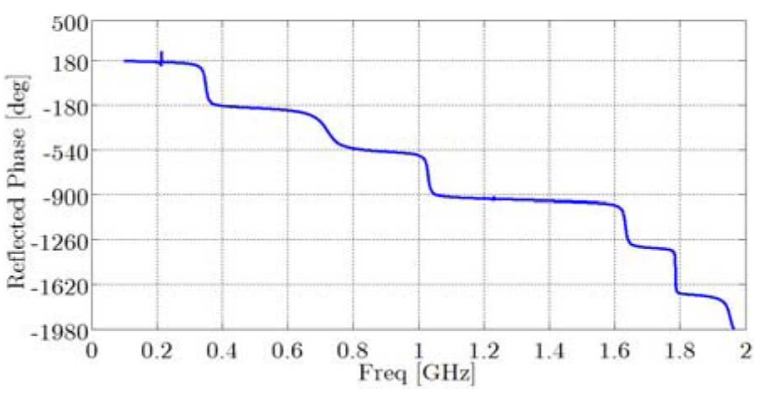

Fig. 10. Reflection phase of the incident wave off the artificial magnetic surface.

\section{INKJET-PRINTED NANOSTRUCTURES ON RF SYSTEMS-ON-PAPER AND WIRELESS SENSORS}

1) Inkjet Printing of Carbon Nanotubes (CNTs): The unique versatility to deposit any solvent-based material by simply changing its ink cartridge, makes inkjet printing particularly low cost and ideally suited in the fabrication of very novel and unique RFID-based sensors with sensing elements embedded in the electrical conductor. This approach could provide the first low-cost "green" and disruptive technology that could bridge the areas of RF, sensing and nanotechnology for the realization of systems-on-paper for cognitive intelligence applications by eliminating many of the more expensive and multistep fabrication processes currently utilized by cleanroom-based photolithography methods for depositing multiple materials on a substrate. Current RFID-based sensors have been focusing on combining RFID antennae and coils along with a discrete IC, that utilize backscattering principles concurrently to empower analog and digital sensing capabilities. Such an approach results in reduced range or a higher turn-on time or an internal battery within the RFID tag that could potentially set numerous challenges in the integration and the packaging of such structures. In this section, we provide a novel approach of eliminating the IC and using the sensing element and its integration with antenna to convey the sensed data wirelessly in a near-"zero-power" fashion. A battery-free conformal CNT-based RFID-enabled sensor node for gas sensing applications, fully printed directly on paper substrate using the inkjet printing technique is presented as a proof-of-concept.

CNT composites have been found to have electrical conductance values that are highly sensitive to extremely small quantities of gases, in addition to the fact of being compatible with inkjet-printing [9]. Previous CNT-related sensing research has been restricted to RFID tags operating at DC or LF frequency ranges due to instabilities as a result of molecular network formation among the inkjet-printed CNT particles at nanoscale [10]. To increase the frequency of the CNT-based RFID tag up to the free Industrial Scientific and Medical Bands (ISM) at UHF frequencies, a special recipe was developed compatible with the Dimatix Materials Printer DMP-2800.

The P3-Type of Single Walled Carbon nanotubes (SWCNT) was chosen due to its increased dispersion in solvent requiring $0.4 \mathrm{mg}$ or higher per $\mathrm{ml}$ before it aggregates in solvents. A DMCLCP-11610 printer head was used to eject the SWCNT ink droplet onto a flexible substrate. In order to prevent clogging of the printer head, the sample SWCNT powder was dispersed in

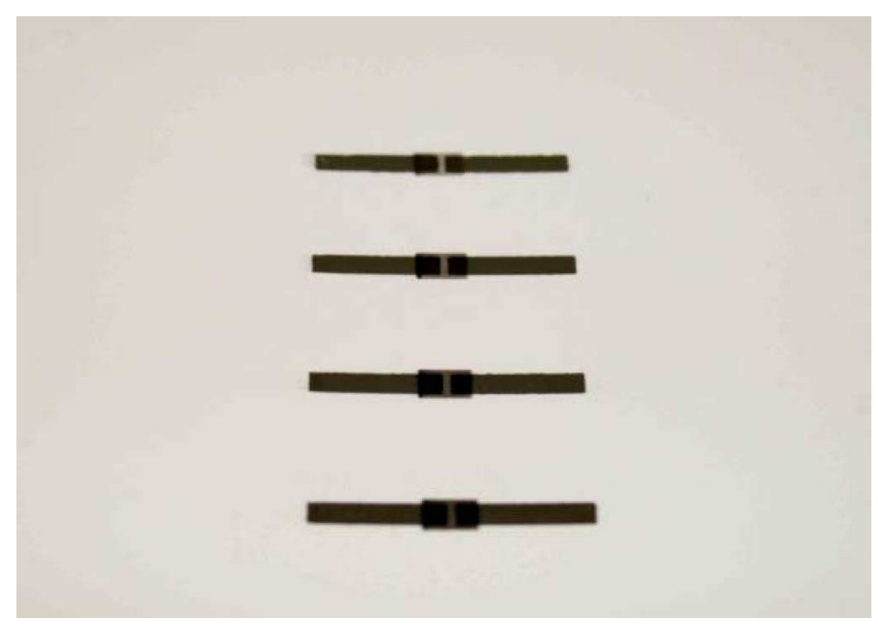

Fig. 11. Photograph of the inkjet-printed SWCNT films with silver electrodes. The number of SWCNT layers of the samples from up to down are 10, 15, 20, and 25, respectively. The dark region shows the overlapping zone between the SWCNT and the silver electrodes.

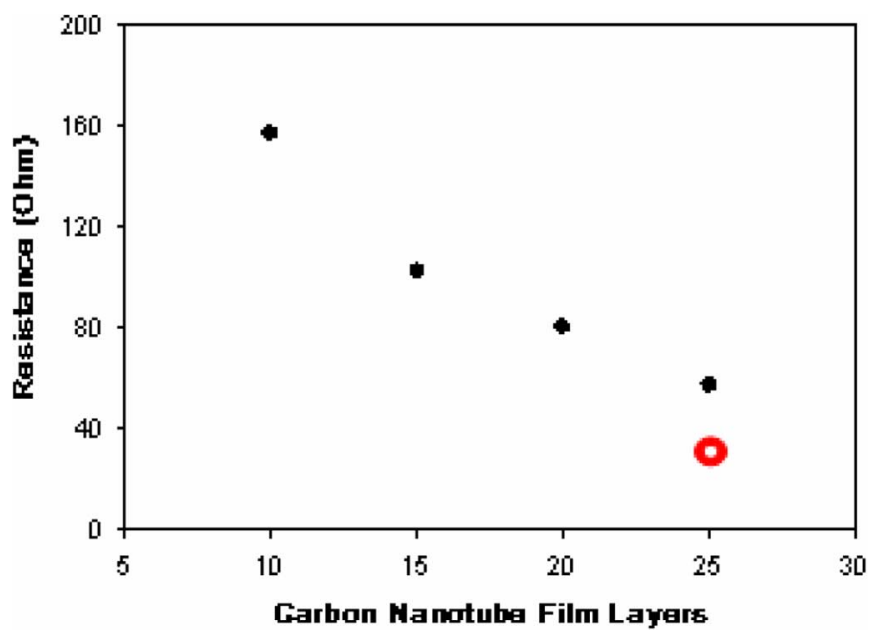

Fig. 12. Measured DC resistance of SWCNT gas sensors in air. Red dot: Resistance of SWCNT device when printed with 1016 dpi setting.

Dimethyl-Formamide (DMF), a polar aprotic solvent and sonicated for $12 \mathrm{~h}$ to prevent aggregations of large particle residues. The concentration of the ink was $0.4 \mathrm{mg} / \mathrm{ml}$ to maximize the concentration of SWCNT within the sprayed solvent for improved sensor sensitivity.

Silver electrodes were patterned with commercial silver-nanosphere ink from Cabot before depositing the SWCNT film, followed by a sintering process. The electrode strip is $2 \mathrm{~mm}$-by- $10 \mathrm{~mm}$ with a gap of $0.8 \mathrm{~mm}$. Then, the $3 \mathrm{~mm}$-by-2 mm SWCNT film was deposited. The $0.6 \mathrm{~mm}$ overlapping zone is to ensure the good contact between the SWCNT film and the electrodes. Four devices with 10, 15, 20 , and 25 SWCNT layers were fabricated to investigate the electrical properties, as shown in Fig. 11. The color depth of the SWCNT film is due to the different layers.

The electrical resistance of the device was probed at DC as a function of the number of the printed SWCNT layers and showed a decreasing resistance trend with the addition of more layers as shown in Fig. 12. This resistance can be further reduced by printing higher dots per given area (dpi) for example, 


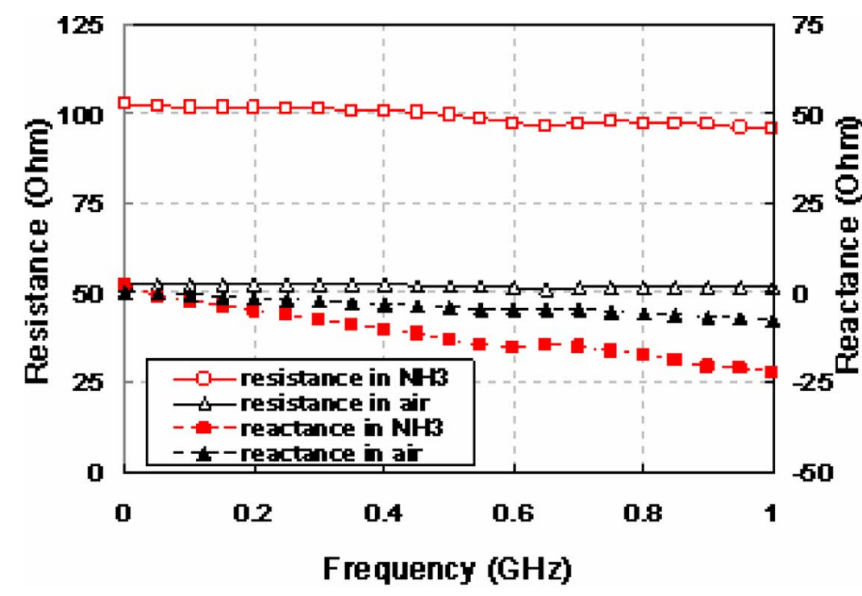

Fig. 13. Measured impedance characteristics of SWCNT film at UHF band.

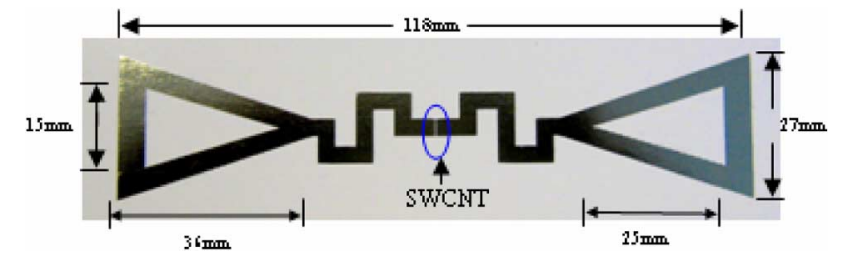

Fig. 14. Inkjet printed RFID sensor tag prototype embedded with SWCNT film on a flexible paper substrate.

the DC resistance drops down even further 20 Ohms using a 1016 dpi setting, as marked by the red circle in Fig. 12. The response of the sensor to different gases such as ethanol, methanol and acetone were also observed in addition to ammonia. For example, the measured value of a 20-layer CNT strip in the first case increased from 80 to $100 \mathrm{Ohms}$, indicating CNTs sensing the presence of the gas.

A network vector analyzer (Rohde\&Schwarz ZVA8) was used to characterize the SWCNT film electrical performance at UHF band before and after the gas reaction. A GS probe was placed on the silver electrodes for the impedance measurements. The calibration method used was short-open-load-thru (SOLT). In Fig. 13, the gas sensor of SWCNT composite shows a very stable impedance response up to $1 \mathrm{GHz}$, which verifies the effectiveness of the developed SWCNT solvent recipe. At $868 \mathrm{MHz}$, the 25-layer sensor exhibits a resistance of $51.6 \Omega$ and a reactance of $-\mathrm{j} 6.1 \Omega$ in air. After meeting ammonia, the resistance was increased to $97.1 \Omega$ and the reactance was shifted to $-\mathrm{j} 18.8 \Omega$. [11]

2) Inkjet-Printed RFID-Enabled CNT-Based Wireless Sensor Tag: With the SWCNT impedance response showing a distinct variability in the presence of gases, a complete RFID tag incorporating this technique was developed. As a final system-level implementation, an RFID-enabled CNT-based Wireless sensor tag was developed in which the silver electrodes connected to the CNT film, as shown in Fig. 14, were inkjet-printed in the form of a specially designed antenna. The operation of the proposed CNT-based sensor utilizes the same backscattering principles that are currently in use with existing RFIDs in order to communicate the data from the tag side in a passive configuration. Unlike the logic-altered impedance of the antenna in a conventional RFID tag, the CNT-based wireless sensor tag utilizes the CNT to alter the antenna impedance in the presence of

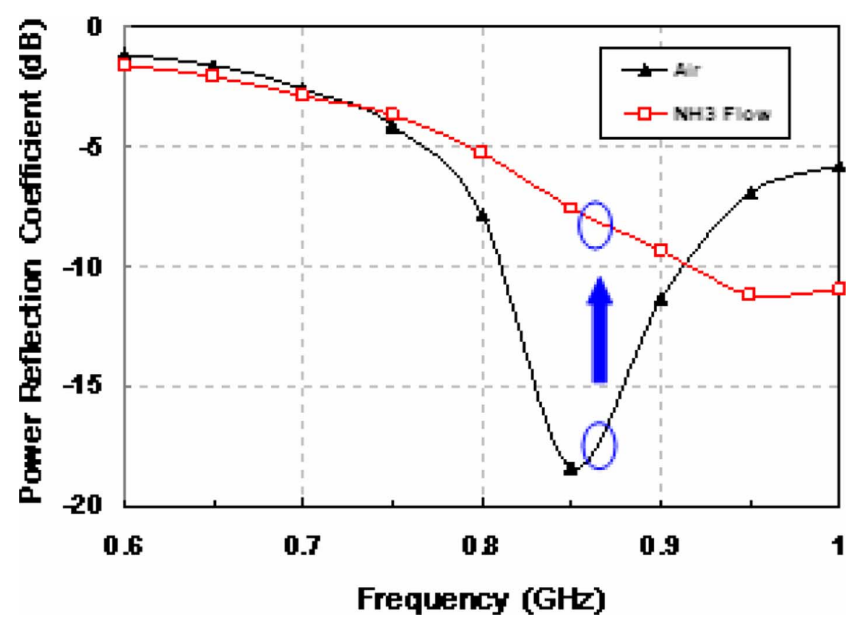

Fig. 15. The calculated power reflection coefficient of the RFID tag antenna with a SWCNT film before and after the gas flow.

a chemical gas, thus altering the amount and the frequency of the incident power from the reader that is reflected back. This proportion represent by the power reflection coefficient of the antenna $(\eta)$ is given by (1)

$$
\eta=\left|\frac{Z_{\text {Ioad }}-Z_{A N T}^{*}}{Z_{\text {load }}+Z_{A N T}}\right|^{2}
$$

where $Z_{\text {Load }}$ represents the impedance of the load and $Z_{\mathrm{ANT}}$ represents the impedance of the antenna terminals with $Z_{\mathrm{ANT}}^{*}$ being its complex conjugate. The same mechanism can be used to realize RFID-enabled sensor modules. The SWCNT film functions as a tunable resistor $Z_{\text {Load }}$ with a value determined by the existence of the target gas. The RFID reader monitors the backscattered power level. When the power level (controlled by the modification of the imaginary part of the CNT-based impedance) and/or the frequency (controlled by the modification of the real part of the CNT-based impedance) changes, it means that there is variation in the load impedance, therefore the sensor detects the existence of the gas. Calculated from Friis free-space formula, the backscattered power in decibels received by the RFID reader is defined as (2) [12]

$$
\begin{gathered}
P_{R}=P_{T}+2 \cdot G_{T}+2 \cdot G_{R}-40 \cdot \log _{10}\left(\frac{4 \pi}{\lambda}\right) \cdots \\
\cdots-40 \cdot \log _{10}(d)+\eta
\end{gathered}
$$

where $P_{t}$ is the power fed into the reader antenna, $G_{t}$ and $G_{r}$ is the gain of the reader antenna and tag antenna, respectively, and $d$ is the distance between the reader and the tag. In (2), except the term of $\eta$, all the other values remain constant before and after the RFID tag meets gas. Therefore, the variation of the backscattered power level solely depends on $\eta$, which is determined by the impedance of the SWCNT film.

The proposed RFID enabled CNT-based Wireless Sensor Tag prototype and its power reflection coefficient in air and ammonia gas are shown in Figs. 14 and 15, respectively.

3) CNT Loaded Antenna-Based Ammonia Gas Sensor: Specially functionalized CNTs have been integrated with antenna 


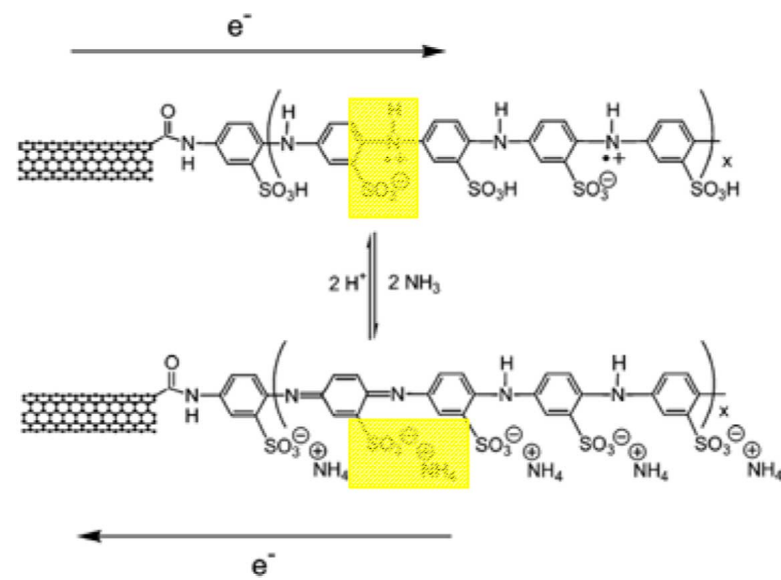

Fig. 16. Mechanism of interaction of PABS-SWNT with $\mathrm{NH}_{3}$. The arrows indicate charge transfer between SWNT and PABS (Bekyarova et al. [13]).

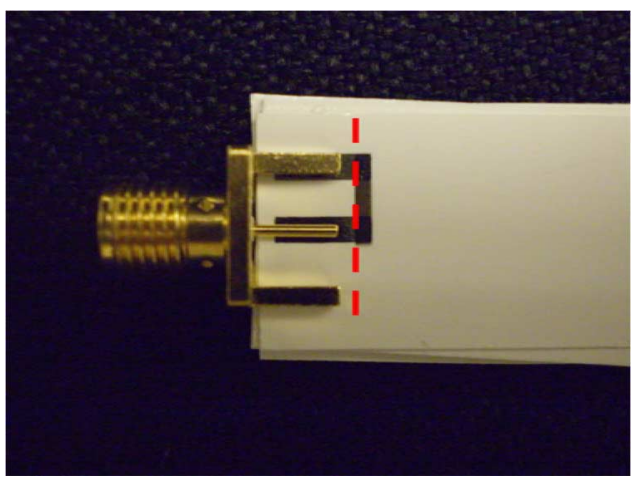

Fig. 17. CNT film placed at the edge of printed silver lines.

design to create a highly sensitive, antenna-based, "smart skin" gas sensor that has the dual function of both sensing and communicating, and that is completely inkjet printable on paper substrate. The functionalized SWCNT will be introduced, followed by its electromagnetic characterization. Then, based on the characterization results, the sensor design is explained followed by closed-system measurement setup and results.

The poly-aminobenzene sulfonic acid (PABS)-SWCNT, shown in Fig. 16, has a polymer that coats the nanotube. The polymer attracts $\mathrm{NH} 3$ and creates a $\mathrm{NH} 4$ bonding, where the ammonia gas particle is physically attached to the SWNT. This is also hydrophilic, which allows it stay well suspended in water solution for inkjet printing purposes [13].

This PABS-SWCNT is mixed with water and sonicated for $2 \mathrm{~h}$, after which it is left over night to observe suspension stability.

Characterization of a thin film of PABS-SWCNT on paper substrate is necessary to know its EM properties and, have a model for PABS-SWCNT, that can be used in a sensor-based design. The thin film PABS-SWCNT was inkjet printed on a short section of transmission line made up of inkjet printed silver nano ink, which was fed using a SMA type feed, as shown in Fig. 17. The effect of the feed lines right upto the PABS-SWCNT denoted by the red line in Fig. 17 were de-embedded using, a Thru-Reflect-Line (TRL) type calibration set that was created by printing a set of Short, Open, Through, and Load transmission line coupons with silver nano-ink.

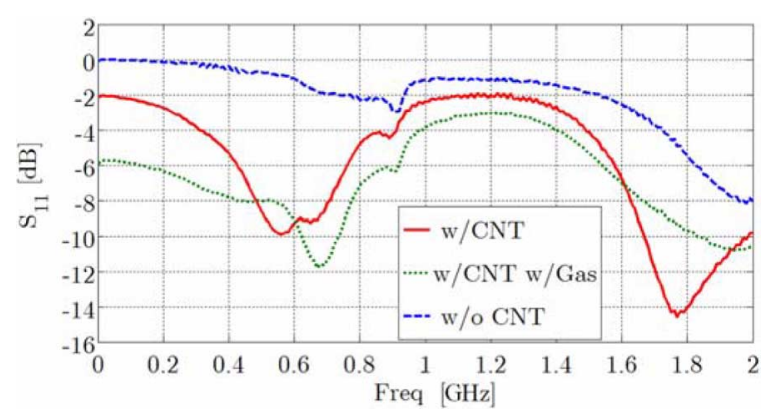

Fig. 18. The input reflection coefficient at the connector coaxial feed in different scenarios (50 ppm ammonia concentration).

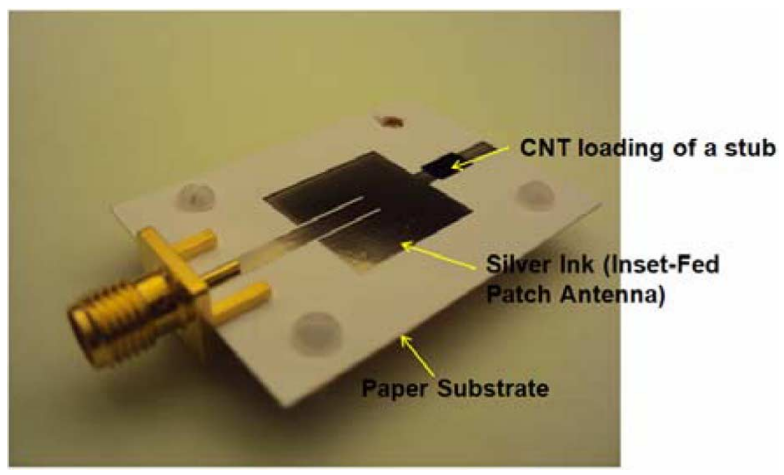

Fig. 19. The RFID tag module design on flexible with inkjet-printed SWCNT film as a load. [15].

From the TRL extraction, the $Z$-parameters of the PABSSWNT were derived and the resistance and reactance values were used as the CNT model [14]. From those values, a parallel $\mathrm{RC}$ circuit was created as the impedance model. It is important to note that the resulting equivalent resistance and capacitance can be designed to a desired value by altering the dimensions of CNT thin film (width and length), and its concentration (or thickness).

The characterization of paper was been done in previous work and the dielectric constant and loss tangent was used for the sensor design [15]. Measurement was conducted on three different scenarios, as shown in Fig. 18. There is a clear resonance shift of S11 with the presence of ammonia gas on the PABS-SWNT.

Given the impedance model of the PABS-SWNT film, the antenna was designed based on several design considerations. The inkjet-printed SWNT behaved as a lossy conductor, so it was isolated from the antenna by introducing it as a load. Second, maximum wireless read range was desired so a patch antenna was used for high directivity. The finalized sensor design was an inset-fed patch antenna with a loading stub onto which the PABS-SWNT was printed, as shown in Fig. 19. For a controlled measurement setup, a $491 \mathrm{M}$ gas generator and $\mathrm{NH}_{3}$ permeation tube was used to control the ammonia gas concentration. The sensor was installed in a closed test cell and a PNA-L N5230A programmable network analyzer (PNA), and data acquisition system was used to measure the $S$-parameters and acquire the data at certain time intervals. At all times, this system was used under a chemical fume hood. 


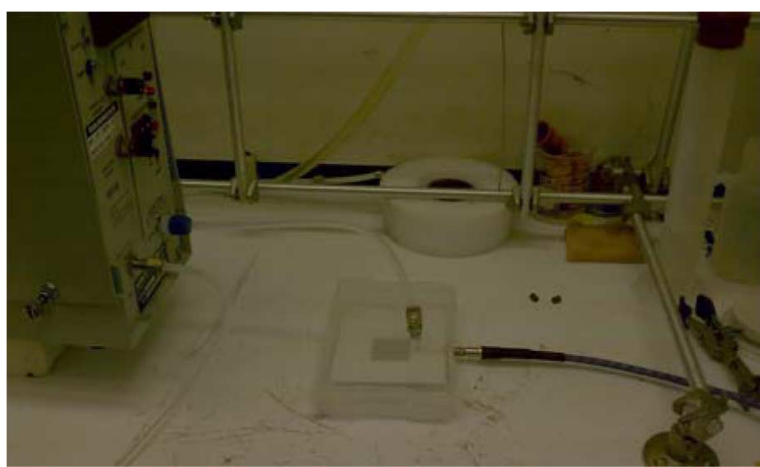

Fig. 20. The calculated power reflection coefficient of the RFID tag antenna with a SWCNT film before and after the gas flow.

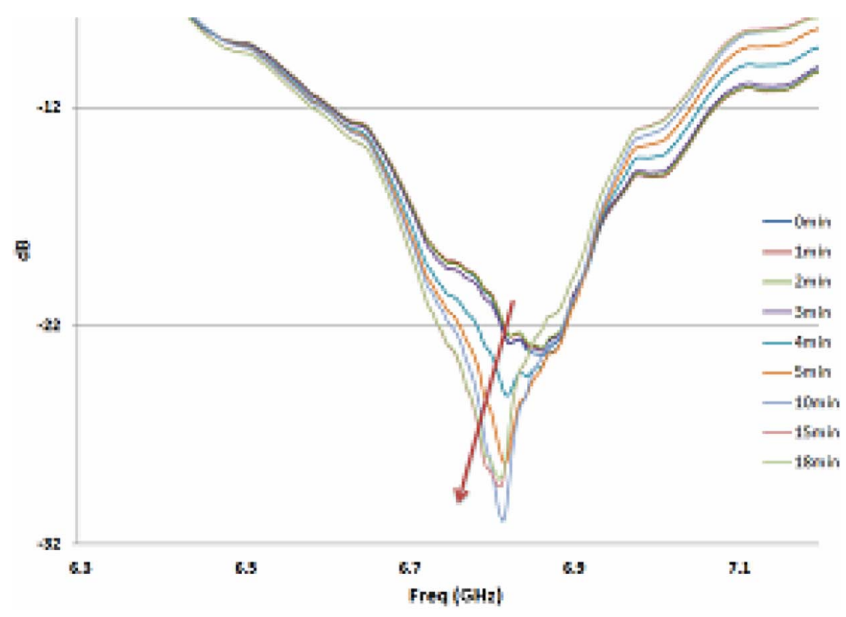

Fig. 21. The resonance peak of the return loss shifts to lower frequencies over time.

Next, the effects of the antenna return loss $\left|S_{11}\right|$ were observed at three different concentrations: 50, 75, and $100 \mathrm{ppm}$. For each measurement, the data was saved automatically every 10 s. Each measurement was conducted by controlling the flow rate which, in turn, controls the ammonia concentration. The sensor was exposed for 18 minutes and then the ammonia was flushed out of the closed test cell with nitrogen at the reversion flow rate using the test setup shown in Fig. 20.

The shift in the resonance of the sensor return loss in decreasing concentrations of ammonia as captured by a vector network analyzer is shown in Fig. 21. The frequency shift as a function of ammonia concentration is plotted in Fig. 22 and shows that higher concentration yields larger resonant frequency shift. Also, the plot shows a decreasing amount of shift for incrementally higher concentrations past $80 \mathrm{ppm}$ level, indicating a trend towards saturation of the sensor with ammonia. The results of the tag response to the incident gases are summarized in Table I.

\section{RF CERTIFICATE OF AUTHENTICATION (RF-COA): \\ A NOVEl INKJET_PRINTABLE RFID SYSTEM WITH A HARDWARE-BASED AUTHENTICATION FEATURE AT 5-6 GHz}

\section{A. RF COA Reader Design and Implementation}

The excellent accuracy $(\sim 50-100 \mu \mathrm{m})$ and conductivity features of the presented inkjet printing process on paper led to the development of a very novel RFID system operating in the free

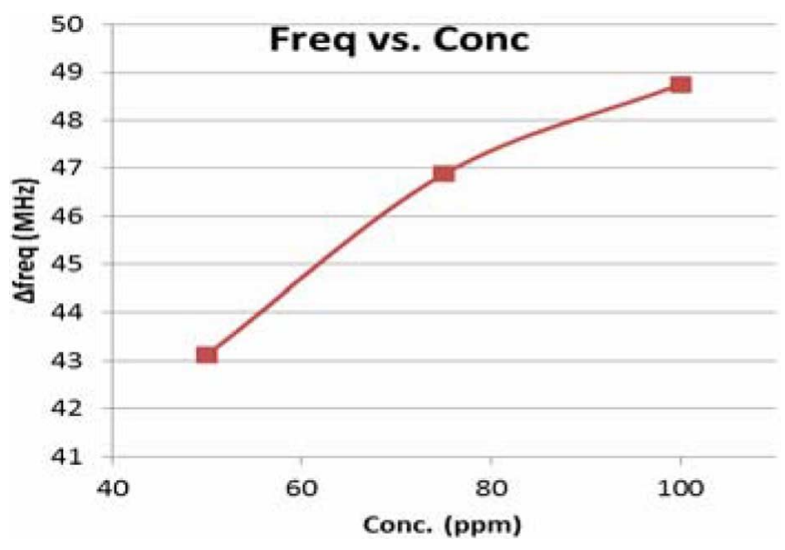

Fig. 22. Measurement plot of resonant frequency shift versus concentration of $\mathrm{NH}_{3}$ gas.

TABLE I

MEASUREMENT RESUlTS: SENSOR $\left|S_{11}\right|$ FREQUENCY SHIFT FOR DifFERENT CONCENTRATIONS OF AMMONIA GAS

\begin{tabular}{|ccc|}
\hline $\begin{array}{c}\text { Conc. Ammonia } \\
(\mathrm{ppm})\end{array}$ & $\begin{array}{c}\text { Exposure } \\
\text { Flow Rate } \\
(\mathrm{L} / \mathrm{min})\end{array}$ & $\begin{array}{c}\Delta \text { fres of S11 } \\
(\mathrm{MHz})\end{array}$ \\
\hline 50 & 0.46 & 43.125 \\
75 & 0.31 & 46.875 \\
100 & 0.23 & 48.750 \\
\hline
\end{tabular}

band at SHF (5-6 GHz) fully realizing a hardware-based authentication function. Conductivity of the silver ink varies between $0.4 \sim 2.5 \times 10^{7} \mathrm{~S} / \mathrm{m}$ depending on curing temperature and time [1] making it very close to copper and aluminum that are commonly used conductors at SHF. Unlike traditional RFIDs with encoded digital information, the proposed solution is to use inkjet-printed diversified tags that have a certain distinctive electromagnetic signature in response to an incident electromagnetic field oscillating at the full 5-6 GHz. This EM signature, that is unique to each tag, constitutes its certificate of authenticity (RF-CoA) [17].

Each of these RF-COAs is a random constellation of small, randomly 3D-shaped conductive and/or dielectric objects that exhibits a distinct behavior in its near-field when exposed to RF waves over a particular RF spectrum in the free SHF bands. The conductive reflectors on these RF-COAs are closed shapes that behave as closed-loop resonators or linear back radiators of the incident field.

The folded shape was chosen for dimensional miniaturization and density maximization. The average loop circumference is $18.6 \mathrm{~mm}$, approximately between half wavelength and one wavelength in size at around $5 \mathrm{GHz}$. Multiple such RF-COAs are shown in separate layers in Fig. 23(b) before they are tightly stacked to give the projection, shown in Fig. 23(a). Another RF-COA design that was also used for the experiments consists of a random constellation of $1 \mathrm{~mm}$-by- $1 \mathrm{~mm}$ pixels that trace the form of the final geometry, shown in Fig. 23(c)

The RFID reader used to read the EM signature of these uniquely patterned RF-COA tags is shown in Fig. 24. It comprises of 5-by-5 antenna matrix of 25 individual microstrip patch elements. Nine of the patches operate as transmitter to illuminate the RF-COA placed within the readers near field, while 


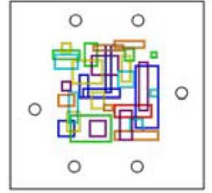

a.

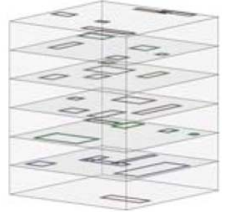

b.

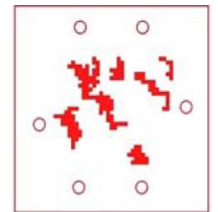

c.
Fig. 23. (a) Single layer COA of rhombic loops. (b) 3D-stacked RF-COAs of rhombic loops. (c) RF-COA as a random trajectory of pixels.

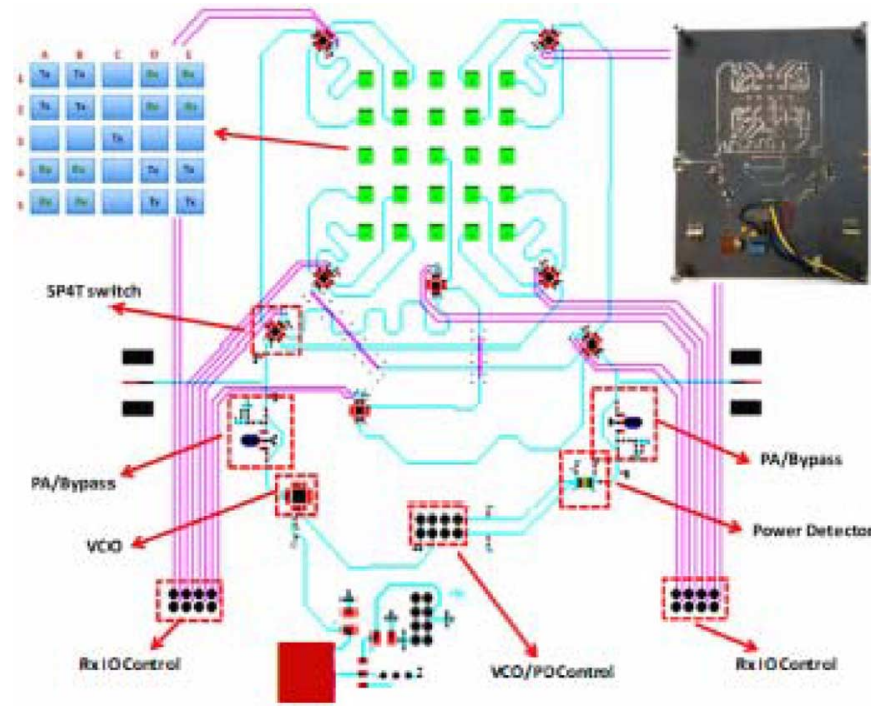

Fig. 24. Circuit layout of the RF-COA reader and its first fabricated version.

eight of patches are used as receivers to read the reflected EM signature from it [17].

The RF-COA reader [17] shown in Fig. 24, extracts the EM signature of an RF-COA instance by measuring and recording a set of gain or S21 parameters for a subset of or all possible antenna couplings that form the array. The measurements are carried out from 5-6 GHz. The scattered, reflected and refracted signal received is amplified by a Power Amplifier and the RF power is monitored by a Power Detector (PD). The EM signature is represented as the voltage proportional to the reflected power from the RF-COA obtained over a frequency sweep between 5 and $6 \mathrm{GHz}$, which would be different for each of the RF-COAs

Large-scale, efficient reel to reel processing possible with inkjet printing on paper mentioned before combined with the high level of accuracy this process provides made it a compelling choice for fabricating the RF-COAs. A prototype of the inkjet printed resonators on paper that form the RF-COAs are shown in Fig. 25. The smallest thickness of the reflectors printed was $200 \mu \mathrm{m}$.

Measurement results in the form of the voltage output proportional to the reflected power from different inkjet printed RF-COAs are shown in Fig. 26. While the patterns of the resonators themselves do affect the reflected power, noticeable resonances were seen between 5.37 and $5.57 \mathrm{GHz}$ for the different RF-COAs. For optimally shaped patterns such as COA6 and COA5, resonances with significantly higher Q-factor were
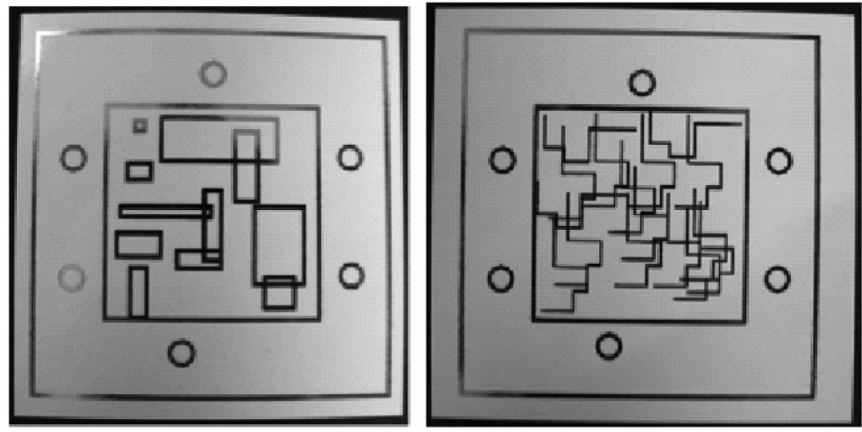

Fig. 25. Inkjet printed RF-COAs.

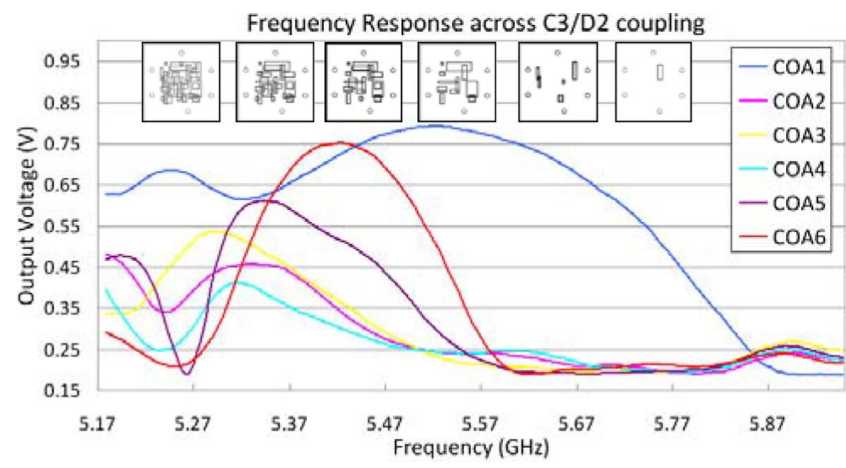

Fig. 26. Effect of the conductive material density on frequency response across $\mathrm{Tx} / \mathrm{Rx}$ coupling at C3/D2 with six COAs with COA1 being the densest and COA6 the sparsest.

observed around 5.37 and $5.47 \mathrm{GHz}$ that suggest significant reflected power at these frequencies with relatively low loss through the inkjet printed resonators. Substantial amounts of power reflection can be seen even with RF-COA having the sparsest of inkjet printed resonators (COA6) across the frequency band between 5-6 GHz. While the working results of the RF-COA RFID system demonstrate the feasibility of the inkjet printing technique and frequencies higher than UHF, additional work to optimize the process for reducing the loss even further are ongoing.

\section{Ambient Powered Wireless SENSOR Modules: BATTERY, SOLAR, AND RF POWERED RFID TAGS}

It is well known that one of the major packaging challenges of RF/Wireless sensors is their efficient and "rugged" integration with power sources. In this section, various wireless delivery platforms to convey the sensed data are presented. Power considerations play an important role for logistical or environmental reasons. By the use of extremely low-power sleep modes and limited operating duty cycles of the wireless sensors; their operable lifetimes can be increased to years on a single coin cell. Increasing the operating lifetime and wireless range of such sensors require careful codesign of the wireless front end including the antenna, embedded technology and its power interface. In this section, the authors present two different wireless sensor platforms that utilize battery and energy harvesting techniques to carry out a temperature-based sensing function over long ranges and lifespan. 


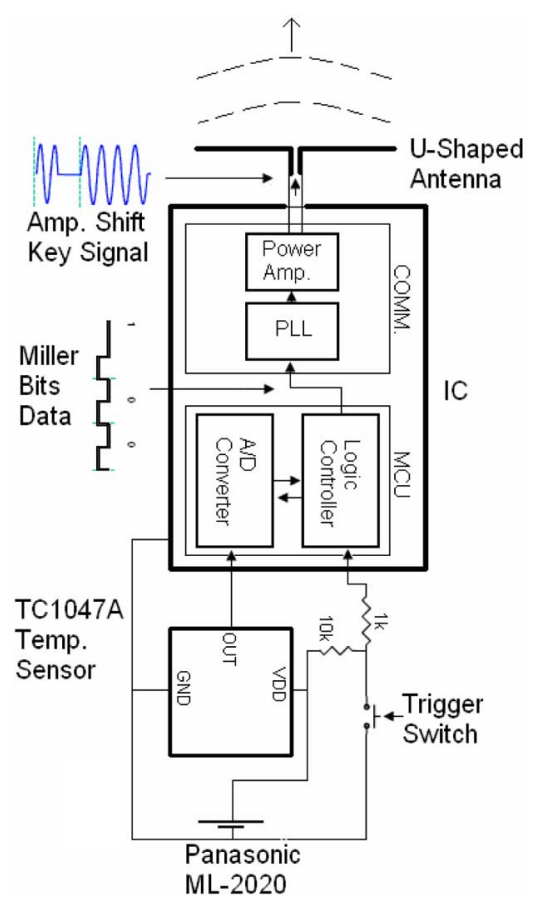

Fig. 27. System level diagram of the paper-based wireless sensor modules.

\section{A. Active Paper-Based RFID Tag for Temperature Sensing}

To investigate the feasibility of paper as a high-frequency substrate for battery powered active RFID application, a microcontroller enabled wireless sensor module was realized on a photo-paper-based substrate. The system-level diagram of this wireless transmitter can be seen in Fig. 27 [6].

The microcontroller unit in the system samples the reading out of an analog sensor such as a temperature sensor, and bit encodes its digital form obtained with the help of an integrated analog to digital converter and the analog signal into full 2 subcarrier cycle Miller bits. As a final step, the MCU modulates the power amplifier (PA) in the integrated transmitter module in the same sequence as bit encoded, digital sensor data thereby transmitting out an Amplitude Shift Key modulated signal from the antenna. The transmission frequency of $904.4 \mathrm{MHz}$ was generated by using a crystal oscillator that was tied to the input of the phase locked loop (PLL) unit of the transmitter. The data transmission was to be carried out at the unlicensed UHF frequencies around $900 \mathrm{MHz}$. In order to maximize the range of the wireless sensor, the integrated PA was characterized using a mechanical load pull system in order to determine its most optimum impedance at which the longest possible range for a fixed battery powered supply can be achieved. This optimum impedance looking out of the PA was determined at three different frequencies within the RFID bandwidth in the U.S. and Europe. The center frequency impedence of about $60-\mathrm{j} 74 \mathrm{ohms}$ [7] was used to conjugately match the antenna for acquiring the maximum range.

A half wavelength dipole was chosen as the antenna structure for the wireless sensor module due to its radiation resistance of $75 \mathrm{ohms}$ [19], which is close to the real part of the optimum load impedance $\left(Z_{\mathrm{L}-\mathrm{opt}}\right)$ looking out of the $\mathrm{PA}$ at $904.4 \mathrm{MHz}$ making for an easier impedance match. To ensure an impedance

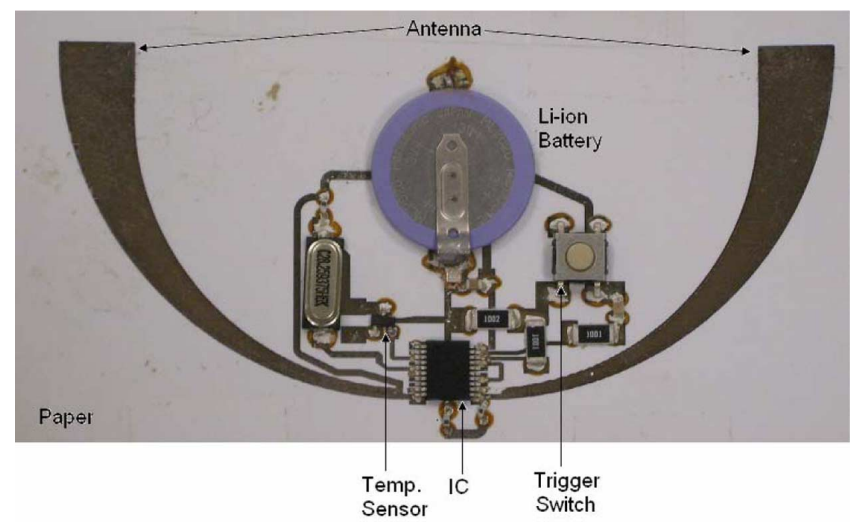

Fig. 28. Active RFID-based wireless sensor module on paper substrate using inkjet printing technology [4].

match, the antenna was optimized so that the impedance looking out of the PA would be close to its optimum value $\left(Z_{\mathrm{L}-\text { opt }}\right)$ at 904.4 MHz (60.1-j73.51 ohms) with the sensor module circuitry connected within the space between the antenna arms. The antenna was printed as a folded dipole on a single layer of a paper substrate with its arms bent around the active sensor circuitry to make the wireless sensor module compact in size.

The proposed circuits for the wireless sensor module including the antennas and circuit layouts were designed and simulated using Ansoft's High-Frequency EM structure simulation (HFSS) software, and printed onto the paper-based substrate using fabricated using A Dimatix DMP-2800 inkjet printer. To ensure maximum conductivity and antenna efficiency, the entire circuit was printed over with 12 layers of silver ink resulting in a conductor thickness of 12 microns [7]. A higher precision inkjet cartridge spraying 1 picoliter droplets was used to print finer areas in the circuit layout such as the IC traces and the PA interface to the antenna ( $\sim 150$ microns), and a 10 Picoliter cartridge was used to print larger areas on the antenna and the RF ground planes/traces in the circuit layout to further increase conductivity. This was done to minimize the gap between adjacently sprayed silver particles enabling a larger overlap during the annealing process and ensuring better conductivity of the printed structure as shown previously to its maximum achievable range between $0.4 \sim 2.5 \times 10^{7}$ Siemens $/ \mathrm{m}$.

The discrete IC components were mounted on the inkjet printed circuit layout using the two-step process shown in Fig. 7. The fabricated battery powered RFID tag can be seen in Fig. 28 [7]. To evaluate its performance wireless link measurements were carried out using a Tektronics RSA 30408A real-time spectrum analyzer (RTSA) connected to a UHF RFID Reader antenna as an RFID reader. The wireless signal emitted by the RFID sensor tag as captures by the RSA is shown in Fig. 29. A minimal shift of only $0.2 \mathrm{MHz}$, was seen from the intended center frequency showcasing the minimum parasitic introduced by the inkjet printed traces. A measured wireless power of $-48.1 \mathrm{dBm}$ was obtained at a distance of 4.26 meters, which would yield a maximum range of 142 and 175 meters with a conventional RFID receiver depending on their relative location [7]. The ASK modulated sensor information sent out by the module at different temperatures that was measured by 


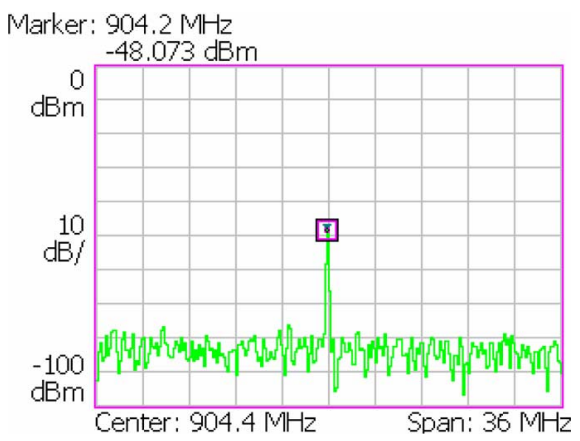

Fig. 29. RTSA measured ASK modulated signal for the dipole-based module from a distance of 4.26 meters. (Power versus frequency.)

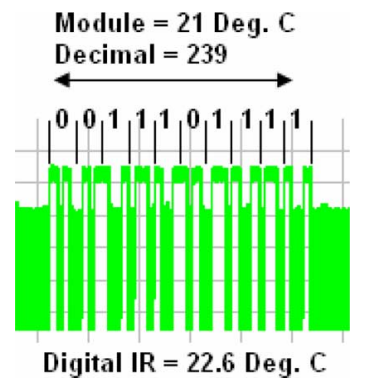

Fig. 30. ASK modulated temperature sensor data captured by the RTSA at room temperature. (Power versus time.)

the RTSA is shown in Fig. 30. The transmitted sensor data shows good agreement with the measurements carried out with the digital infrared thermometer that has an accuracy of $\pm 2.5{ }^{\circ} \mathrm{C}$ thereby showcasing the feasibility of inkjet printing technology towards RF-based sensing platforms in the UHF range.

\section{B. Battery-Less Solar Powered Tag for Health Monitoring}

In [22], the authors have shown a technique that is conventionally used in passive RFID systems towards achieving longer distance wireless link. The technique involved powering on a wireless transmitter and microcontroller unit for limited duty cycles using a highly efficient Ga-As-based solar cell coupled with an in-house built power management unit that encompassed a super capacitor to collect and store the solar energy. Most other solar powered devices utilize a solar cell combined with a DC-DC converter to power on the end device in a continuous manner. Such a mechanism requires a large enough solar cell that can collect enough incident light to continuously power on both the converter and the end device. The presented tag is different in that it uses the solar power intermittently to charge and hold it across a super capacitor, which under the control of a voltage supervisor undergoes a controlled discharge and in the process wake up and power the microcontroller unit (MCU) and wireless transmitter in the tag. Such a mechanism can get away with a much smaller sized solar cell albeit only by communicating asynchronously similar to a passive RFID tag but over significantly longer range. The solar powered tag communicates asynchronously with wireless Mica Mote receivers made by Crossbow [21], which utilizes the low-power, sub-1 GHz CC-1000 transceiver

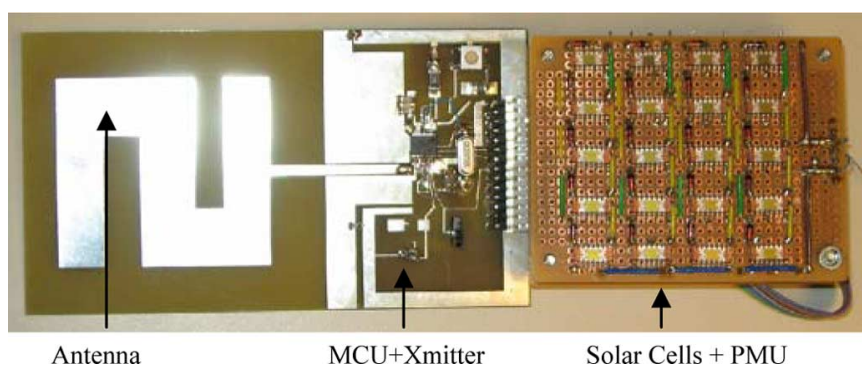

Fig. 31. Battery-less solar powered tag development prototype to test out the solar powered and triggered wireless link.

chip by Texas Instruments (TI). The TI cc-1000-based reader platform consumes much less overall power then conventional RFID readers while syncing more amounts of data at higher range. Range in excess of 500 feet was achieved through the proper characterization of the most power consuming element in the system, PA in the wireless front end with respect to its RF impedance and power consumption profile. Such a solar powered radio that was previously designed for long range RFID-based localization and tracking was modified for use in health care-based bio-monitoring that have less stringent requirements on data payload and operable range but require more frequent sensing and wireless polling. The modified prototype of the solar powered tag is shown in Fig. 31. It utilizes a modified Power Management unit that comprises a super capacitor to collect and store the solar power, an ultra-low-power consuming voltage supervisor, and an optimized microcontroller firmware to control the clock on and off functioning in the MCU in sync with the voltage supervisor. A single payload of sensed temperature data consists of frequency shift key (fsk) modulated preamble bits, Sync ( 2 bytes), data or group length (1 byte), ADC converted temperature sensor read data (16-32 bytes), and CRC (1 byte), as shown in the wireless packets sent out by the tag and measured using a frequency versus time plot on a spectrum analyzer in Fig. 32 [22]. The operable duty cycle during which the wireless front end of the tag requires to stay on is about $9 \mathrm{~ms}$ to broadcast out a single payload once. In order to achieve a coupe of data packet transmissions at a fast enough rate per one charge discharge cycle of the solar energy storing super cap, a low leakage $200 \mu \mathrm{F}$ super capacitor was used in the PMU. The wireless transmission profile of the optimized solar powered tag as measured by a real-time spectrum analyzer (RTSA) used as a receiver is shown in Fig. 33. A $200 \mu \mathrm{F}$ supercapacitor manages to store enough energy to power on and wirelessly transmit data for duration of $30 \mathrm{msec}$, as shown in the power versus time plot in Fig. 33. The wireless transmission was carried out at $904.4 \mathrm{MHz}$ for which the antenna shown in Fig. 31 was optimized.

Super capacitors are much more economical and environmentally cleaner to manufacture. In addition, the number of charge-discharge cycles allowed by supercapacitors are orders of magnitude higher than batteries eliminating the need to replace them every couple of years as required by batteries. Finally, capacitors are much cleaner to dispose of as compared to batteries thereby making such techniques environmentally much friendlier and cheaper. Their only drawback is that they 


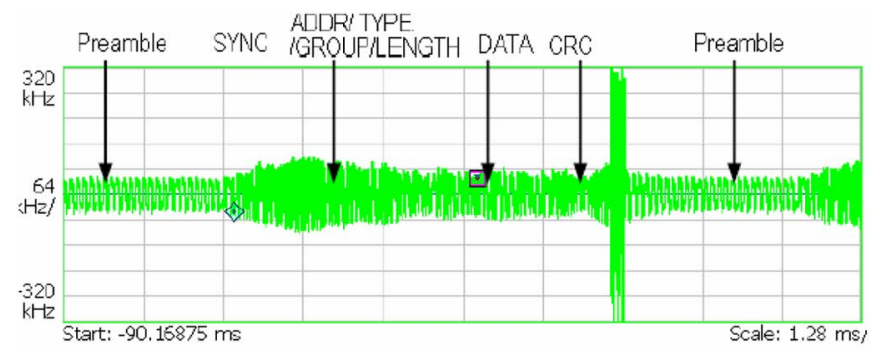

Fig. 32. Wireless data sequence (RFID) sent out by the solar powered tag [15].

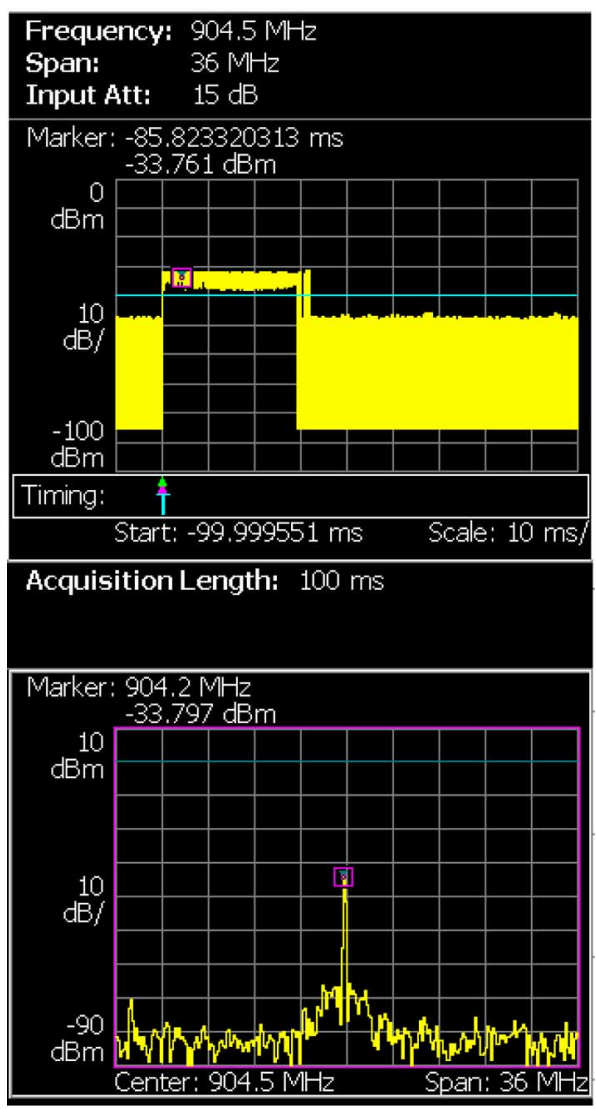

Fig. 33. Wireless radio signal (RFID) sent out by the optimized solar powered tag showing the amount of radio on time as captured by a RTSA.

require a properly designed PMU to maintain an acceptable on and off state for the radio to operate reliably and efficiently.

\section{CONCLUSION}

In this paper, an inkjet-printed "green" system-on-paperbased solution is presented for the ultra-low-cost realization of flexible antennas, RF electronics and sensors The authors briefly touch up the state-of-the-art area of fully integrated wireless sensor modules on paper that could potentially set the foundation for the truly convergent wireless sensor ad hoc networks of the future. Plus, the authors address the unique capability of the proposed approach to bridge nanotechnology, RF, sensing and hardware-enabled authentication for the realization of cognitive intelligence applications. Various challenges of RF packaging, and power sources integration are investigated in terms of ruggedness, accuracy and reliability and flexing performance for space, automotive, "smart-skin" and wearable applications for applications up to $6 \mathrm{GHz}$.

\section{ACKNOWLEDGMENT}

The authors would like to thank the following sponsors for the presented research: the National Science Foundation (NSF), NEDO, and IFC.

\section{REFERENCES}

[1] L. Yang, A. Rida, R. Vyas, and M. Tentzeris, "RFID tag and RF structures on a paper substrate using inkjet-printing technology," IEEE Trans. Microw. Theory Tech., vol. 55, no. 12, pp. 2894-2901, Dec. 2007.

[2] Roger's Corporation, "ULTRALAM ${ }^{\circledR} 3000$ Series Liquid Crystalline Polymer Circuit Materials," 2011.

[3] "LPKF ProtoLaser S datasheet," LPKF Laser and Electronics, AZ, Germany, 1989. [Online]. Available: http://www.lpkf.com/products/rapid-pcb-prototyping/laser-circuit-structuring/laser-structuring-printed-circuit-boards.htm

[4] Dimatix DMP 28XX PC Control Software. Santa Clara, CA: Fujifilm USA Dimatix Inc., 2008.

[5] Dimatix DMP 28XX Data Manual. Santa Clara, CA: Fujifilm USA Dimatix Inc., 2008.

[6] R. Vyas et al., "Paper-based RFID-enabled wireless platforms for sensing applications," IEEE Trans. Microw. Theory Tech., vol. 57, no. 5, pp. 1370-1382, May 2009.

[7] R. Vyas, A. Rida, L. Yang, and M. M. Tentzeris, "Design, integration and characterization of a novel paper based wireless sensor module," in Proc. IEEE Int. Microw. Symp., Jun. 2008, pp. 1305-13078.

[8] G. Shaker, H. Lee, K. Duncan, and M. Tentzeris, "Integrated antenna with inkjet-printed compact artificial magnetic surface for UHF applications," in Proc. IEEE Int. Conf. Wireless Inform. Technol. Syst., Sep. 2010, pp. 1-4.

[9] J.-H. Yun, H. Chang-Soo, J. Kim, J.-W. Song, D.-H. Shin, and Y.-G. Park, "Fabrication of carbon nanotube sensor device by inkjet printing," in Proc. IEEE Nano/Micro Eng. Molecular Syst., Jan. 2008, pp. 506-509.

[10] M. Dragoman, E. Flahaut, D. Dragoman, M. Ahmad, and R. Plana, "Writing electronic devices on paper with carbon nanotube ink," ArXiv-0901.0362, 2009.

[11] L. Yang, G. Orecchini, G. Shaker, L. Hoseon, and M. Tentzeris, "Battery-free, RFID-enabled, wireless sensors," in IEEE Int. Microw. Symp., Jul. 2010, pp. 1528-1531.

[12] J. Seybold, Introduction to RF Propagation. Hoboken, NJ: Wiley, 2005, pp. 111-133.

[13] E. Bekyarova, I. Kalinina, M. E. Itkis, L. Beer, N. Carbrera, and R. C. Haddon, "Mechanism of ammonia detection by chemically functionalized single-walled carbon nanotubes: In situ electrical and optical study of gas analyte detection," J. Amer. Chem. Soc., vol. 129, pp. 10700-10706, 2007.

[14] R. B. Marks, "A multiline method of network analyzer calibration," IEEE Trans. Microw. Theory Tech., vol. MTT-39, no. 7, pp. 1205-1215, Jul. 1991.

[15] J. Kupka, R. N. Clarke, O. C. Rochard, and A. P. Gregory, "Split post dielectric resonator technique for precise measurements of laminar dielectric specimens-measurement uncertainties," Microwaves, Radar, Wireless Commun., vol. 1, pp. 305-308, 2000.

[16] H. Lee, G. Shaker, and M. Tentzeris, "A novel highly-sensitive antenna-based "Smart skin" gas sensor utilizing carbon nanotubes and inkjet printing," in Proc. IEEE Int. Symp. Antenna Propagat., Jul. 2011, pp. 1593-1596.

[17] V. Lakafosis, A. Traille, H. Lee, G. Orecchini, E. Gebara, M. M. Tentzeris, J. Laskar, G. DeJean, and D. Ki- rovski, "An RFID system with enhanced hardware-enabled authentication and anti-counterfeiting capabilities," in Proc. IEEE Int. Microw. Symp., May 2010, pp. 840-843.

[18] UHF Gen-2 System Overview. Dallas, TX: Texas Instruments, 2005.

[19] C. Balanis, Antenna Theory, Analysis and Design, 3rd ed. New York: Wiley.

[20] L. Yang, A. Rida, T. Wu, S. Basat, and M. Tentzeris, "Integration of sensors and inkjet-printed RFID tags on paper-based substrates for UHF 'Cognitive intelligence' applications,' in Proc. IEEE Antennas Propagat. Int. Symp., Jun. 2007, pp. 1193-1196. 
[21] "Crossbow Mica2 mote, TinyOS community forums," Univ. California, Berkeley, CA, 2008 [Online]. Available: http://webs.cs. berkeley.edu/tos/hardware/sensorboard.html

[22] R. Vyas, V. Lakafosis, and M. Tentzeris, "Wireless remote localization system utilizing ambient RF/solar power scavenging RFID tags," in Proc. IEEE Int. Microw. Symp., May 2010, pp. 1764-1767.

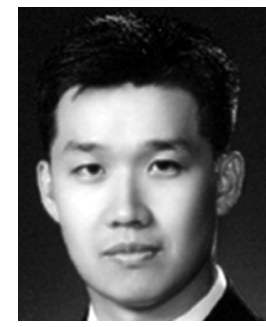

Hoseon Lee received the B.S. and M.S. degrees from the Georgia Institute of Technology, Atlanta, in 2002 and 2005 , respectively. Currently, he is working towards the Ph.D. degree in electrical engineering at the Georgia Institute of Technology.

From 2006 to 2009, he was a naval officer and Assistant Professor with the Republic of Korea (ROK) Naval Academy. His research interests include low-cost organic solutions for radars, communication, sensing and identification systems, inkjet printing, and power scavenging for RF applications.

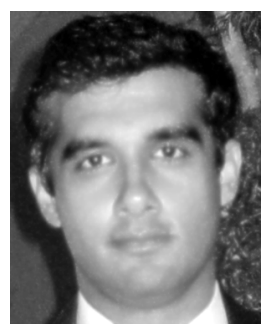

Rushi Vyas (S'06) received the B.S. and M.S. degrees in electrical engineering from the Georgia Institute of Technology, Atlanta, in 2005 and 2010, respectively, with a background in microwave and wireless circuit design, power electronics and embedded systems. He is currently a Researcher at the ATHENA research group at Georgia Institute of Technology while working towards the Ph.D. degree.

His work so far have involved utilizing a combination of antenna, RF, power and embedded systems for a number of applications. His works in the past have been in areas of antenna design in the millimeter-wave and UHF bands, and development of active and wireless sensor modules on biodegradable organic substrates using inkjet-printing techniques for radar and RFID-based sensing applications. His Ph.D. research work has seen him design and develop several prototypes of energy scavengers that harness ambient power present in sources such as mechanical motion, solar and TV/cellular wireless signals to power on embedded microcontrollers and wireless transceivers. In addition to designing the hardware, he is also responsible for developing the software/firmware that forms part of the power management unit and physical layers of the energy harnessing wireless modules. His energy harnessing wireless prototypes are finding use in a number of applications, namely, wireless triangulation (localization), building structure monitoring and remotely powered wireless sensors. He has authored/coauthored over 20 research papers in peer-reviewed journals and conference proceedings and one book chapter covering topics such as antenna design, wireless/microwave circuit design, low power electronics, wireless sensors and ubiquitous computing. His work on power scavenging has been well received in IEEE APS and IMS conferences and in wider public media forums such as MSN news, Design World, Engadget among several others.

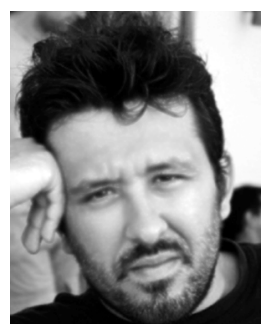

Vasileios Lakafosis (S'07) received the Diploma degree in electrical and computer engineering from the National Technical University, Athens, Greece, in 2006 and the M.Sc. degree in electrical and computer engineering from the Georgia Institute of Technology, Atlanta, in 2009. Currently, he is working towards the Ph.D. degree at the Georgia Institute of Technology.

In the past, he has worked for Microsoft Research, the Cisco Research Center, and the University of Tokyo. His cross-disciplinary research interests range from novel networking protocols in the areas of wireless mobile ad hoc, mesh, and sensor networks to perpetual, ambient RF energy harvesting communication systems, security authentication, wireless localization techniques, distributed computing in delay tolerant and opportunistic networks, and ubiquitous computing applications. He has authored and coauthored two book chapters and over 15 papers in peer-reviewed journals and conference proceedings.

Mr. Vasileios is a member of Association for Computing Machinery (ACM), $\mathrm{HKN}$, and the Technical Chamber of Greece. He serves as reviewer for a number of IEEE journal transactions and conference proceedings.

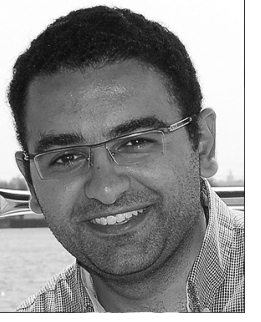

George Shaker (S'99-M'10) received the B.Sc. degree (Hons) in electronics and communications engineering from the University of Cairo, Cairo, Egypt. He studied for the M.A.Sc. and Ph.D. degrees in electrical and computer engineering at the University of Waterloo, Waterloo, ON, Canada.

$\mathrm{He}$ was a Visiting Researcher at the Georgia Institute of Technology, Atlanta (2009/2010). He is currently with the Advanced Electromagnetic Research division of the RF R\&D at Research in Motion. He is also with the Center of Intelligent Antenna and Radio Systems (CIARS) at the University of Waterloo. He has authored/coauthored more than 50 journal publications, patent applications, conference papers, and technical reports. His current research interests are in the areas of antenna synthesis and design, integrated and adaptive front-ends, electromagnetic interference and compatibility, biomedical wireless systems, wireless sensor nodes, printed electronics, and advanced optimization computer-aided design techniques.

Dr. Shaker is a member of the IEEE Antennas and Propagation Society (IEEE AP-S), the IEEE Microwave Theory and Techniques Society (IEEE MTT-S), the IEEE Communications Society, the IEEE Computer Society, and the Applied Computational Electromagnetic Society. He regularly serves as a reviewer for several IEEE and Wiley publications. He has served as an invited speaker as well as a session co-chairman in several international scientific conferences. $\mathrm{He}$ was the recipient of multiple prestigious awards and scholarships including the NSERC Canada Graduate Scholarship (2007-2010), the Ontario Graduate Scholarship (2007-2010), the European School of Antennas Grant at IMSTGmbH (2007), the IEEE AP-S Honorable Mention Paper Award (2008, 2011), the IEEE AP-S Best Paper Award (2009, Third Place), the IEEE Antennas and Propagation Graduate Research Award (2008/2009), NSERC CGS-FSS (2009/ 2010), the IEEE MTT-S Graduate Fellowship (2009), and the Electronic Components and Technology Best of Session Paper Award (2010).

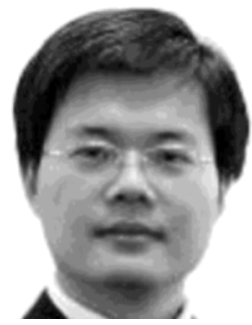

Li Yang (S'04) received the B.S. and M.S. degrees in electronic engineering from Tsinghua University, Beijing, China, in 2002 and 2005, respectively, and the Ph.D. degree in electrical and computer engineering from the Georgia Institute of Technology, Atlanta, in 2009

$\mathrm{He}$ is currently a Research Engineer in the High-Performance Analog Division, Texas Instruments, Dallas. He has published more than 30 papers in refereed journals and conference proceedings, two books, and three book chapters. His research interests include mixed-signal integrated circuit, radio-frequency identification (RFID) technology, and wireless transceivers for sensing and power scavenging applications.

Dr. Yang was the recipient/corecipient of the IEEE Antennas and Propagation Society (AP-S) 2010 Uslenghi Letters Prize Paper Award, the 2007 IEEE AP-S Symposium Best Student Paper Award, the 2007 IEEE International Microwave Symposium (IMS) Third Best Student Paper Award, the 2007 International Symposium on Antennas and Propagation (ISAP) Poster Presentation Award, and the 2006 Asian-Pacific Microwave Conference Award. He is a committee member of the RFID Technical Committee (TC24) of the IEEE Microwave Theory and Techniques Society (MTT-S). 


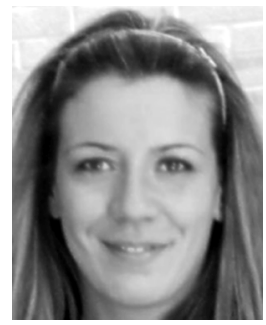

Giulia Orecchini received the Laurea degree in electronic engineering from the University of Perugia, Perugia, Italy, in 2008. Currently, she is working towards the Ph.D. degree in electronic information engineering at the University of Perugia.

In Autumn 2008, she joined the ATHENA Research Group, Georgia Institute of Technology, Atlanta. Her research interests concern the development of RFID electronic systems and technologies.

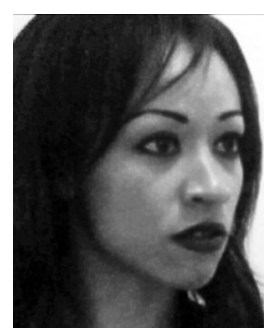

Anya Traille was born in Washington, DC, in 1982 She received the B.S. and M.S. degrees in electrical and computer engineering from the Georgia Institute of Technology, Atlanta, in 2004 and 2009, respectively.

Currently, she is a Research Engineer with the School of Electrical and Computer Engineering, Atlanta, GA. From 2004 to 2007, she was a Full-Time Research Engineer with the Georgia Tech Research Institute, Smyrna. In 2008, she was a Visiting Scholar with Imperial College, London, U.K. In 2009, she was a Visiting Researcher with Georgia Tech Ireland, Athlone. In 2010, she was a Visiting Researcher with LAAS-CNRS, Toulouse, France. Her main areas of research include antenna arrays, waveguides, RFID, and liquid antennas for bio-signal monitoring in which she has organized and chaired numerous conference sessions.

Ms. Traille was the recipient/corecipient of the IEEE IGARSS 2009 Student Travel Award, the ISAP 2007 Best Poster Paper Award, the Second Place Best Paper Award of the Georgia Institute of Technology Graduate Research Fair 2006, and the Best Session Paper Award of IEEE ECTC 2010. She was also a finalist in the 2008 IEEE AP-S Best Student Paper Competition and a finalist in the 2007 ACES Symposium Best Student Paper Competition.

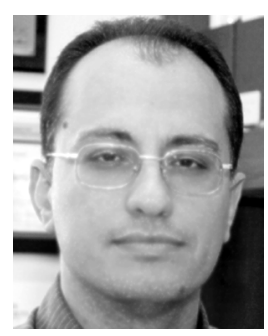

Manos M. Tentzeris (S'89-M'92-SM'03-F'10) received the Diploma degree (magna cum laude) in electrical and computer engineering from the National Technical University of Athens, Athens, Greece, and the M.S. and Ph.D. degrees in electrical engineering and computer science from the University of Michigan, Ann Arbor.

$\mathrm{He}$ is currently a Professor with the School of ECE, Georgia Tech, Atlanta. He has published more than 420 papers in refereed Journals and Conference Proceedings, 5 books and 19 book chapters. He has helped develop academic programs in highly integrated/multilayer packaging for RF and wireless applications using ceramic and organic flexible materials, paper-based RFID's and sensors, "Green" electronics and power scavenging, nanotechnology applications in RF, microwave MEM's, SOP-integrated (UWB, mutliband, conformal) antennas and adaptive numerical electromagnetics (FDTD, multiresolution algorithms) and heads the ATHENA research group (20 researchers). He has served as the Georgia Electronic Design Center Associate Director for RFID/sensors research from 2006 to 2010, and he has been the Georgia Tech NSF-Packaging Research Center Associate Director for RF Research and the RF Alliance Leader from 2003 to 2006. He was a Visiting Professor with the Technical University of Munich, Germany, for the summer of 2002, a Visiting Professor with GTRI-Ireland in Athlone, Ireland, for the summer of 2009, and a Visiting Professor with LAAS-CNRS in Toulouse, France, for the summer of 2010 . He has given more than 100 invited talks to various universities and companies all over the world.

Dr. Tentzeris is a member of URSI-Commission D, a member of MTT-15 committee, an Associate Member of EuMA, a Fellow of the Electromagnetic Academy, and a member of the Technical Chamber of Greece. He is one of the IEEE MTT-S Distinguished Microwave Lecturers from 2010-2012. He was the recipient/co-recipient of the 2010 IEEE Antennas and Propagation Society Piergiorgio L. E. Uslenghi Letters Prize Paper Award, the 2011 Best Student Paper Award at the International Workshop on Structural Health Monitoring, the 2010 Georgia Tech Senior Faculty Outstanding Undergraduate Research Mentor Award, the 2009 IEEE Transactions on Components and Packaging Technologies Best Paper Award, the 2009 E. T. S. Walton Award from the Irish Science Foundation, the 2007 IEEE APS Symposium Best Student Paper Award, the 2007 IEEE IMS Third Best Student Paper Award, the 2007 ISAP 2007 Poster Presentation Award, the 2006 IEEE MTT Outstanding Young Engineer Award, the 2006 Asian-Pacific Microwave Conference Award, the 2004 IEEE Transactions on Advanced Packaging Commendable Paper Award, the 2003 NASA Godfrey "Art" Anzic Collaborative Distinguished Publication Award, the 2003 IBC International Educator of the Year Award, the 2003 IEEE CPMT Outstanding Young Engineer Award, the 2002 International Conference on Microwave and Millimeter-Wave Technology Best Paper Award (Beijing, China), the 2002 Georgia Tech-ECE Outstanding Junior Faculty Award, the 2001 ACES Conference Best Paper Award and the 2000 NSF CAREER Award and the 1997 Best Paper Award of the International Hybrid Microelectronics and Packaging Society. He was the TPC Chair for the IEEE IMS 2008 Symposium and the Chair of the 2005 IEEE CEM-TD Workshop. He is the Vice-Chair of the RF Technical Committee (TC16) of the IEEE CPMT Society. He is the founder and Chair of the RFID Technical Committee (TC24) of the IEEE MTT Society and the Secretary/Treasurer of the IEEE C-RFID. He is the Associate Editor of the IEEE TRANSACTIONS ON MiCROWAVE THEORY AND TECHNIQUES, the IEEE TRANSACTIONS ON ADVANCED PACKAGING, and the International Journal on Antennas and Propagation.

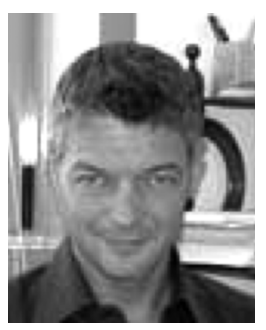

Luca Roselli (M'99-SM'01) received the Laurea degree in electronic engineering from the University of Florence, Florence, Italy, in 1988.

From 1988 to 1991, he worked at the University of Florence on SAW devices. In November 1991, he joined the Institute of Electronics at the University of Perugia, Perugia, Italy, as a Research Assistant. Since 1994, he has been teaching electronic devices at the same University. In the same year, he was the Director of works for the realization of a 35 square meters clean room 100 class at the University of Perugia. Since 2000, he has been an Associate Professor with the University of Perugia, where he teaches microwave electronics, high-frequency components, and applied electronics. Since that time, he has been coordinating the research activity of the High Frequency Electronics (HFE) Lab. In the same year, he founded WiS (Wireless Solutions) Srl, a spin off company operating in the field of microwave electronic systems with which he is currently cooperating as a consultant. Since 2003 he is in the steering committee of CEM-TD (Computational ElectroMagnetic-Time Domain). In the same year, he was a member of the spin off committee of the University of Perugia, in 2005 he founded a second spin off company: DiES (Digital Electronic Solutions) Srl. He is Coordinator of the High Frequency Electronic Lab (HFE-Lab) at the University of Perugia. His research interests mainly focus on the design of high frequency electronic circuits and systems, including the development of numerical methods for electronic circuit analysis with special attention to RFID systems and new material electronics. In these fields he published almost 200 contributions to international reviews and peer reviewed conferences the interest in which is testified by an $\mathrm{HF}$ index of 16 (PoP font) and more than 800 citations on international reviews and peer reviewed conferences.

Prof. Roselli has been a Reviewer of the IEEE MiCROWAVE AND GUIDED WAVE LETTERS (MGWL), now MWCL, since 1996. Since 1998, he has been a Reviewer of the IEEE TRANSACTIONS ON MICROWAVE THEORY AND TECHNIQUES (MTT). Since the same year, he has been a member of the Technical Program Committee of the Electrosoft Conference, and of the MTT-International Microwave Symposium. In 2007, he was the Chairman of the VII Computational Electromagnetic in the Time Domain Workshop. He was the Guest Editor of the Special Issue of the International Journal of Numerical Modelling on the VII CEM-TD. Again in 2008, he was the Co-PI (Principal Investigator) of the Project ADAHELI (project founded by the Italian Space Agency-ASI for the solar observation from the satellite. His main current servicing activities are: member of the list of experts of the Italian Ministry of Research and University (MIUR); member of Technical Committee MTT-25-RF Nanotechnologies; Chairman of Technical Committee MTT-24-RFID Technologies; Chairman of Sub Committee 32-RFID Technologies of IMS; member of the European Research Council (ERC) Panel PE7; Representative of Italy in the COST ACTION IC0803 RF/Microwave Communication Subsystems for Emerging Wireless Technologies (RFCSET); PI for the University of Perugia of the ENAIC Project EnLIGHT; CO-PI for the University of Perugia of the ENIAC Project ARTEMOS; member of the Technical Program Committee of RWCOM Conference; member of the Technical Program Committee of RFID-TA Conference; member of the Steering Committee of "Agenzia Umbria Ricerche" in the framework of POR projects; and Director of the Science and Technology Committee of the research center "il Pischiello." He is Coordinator of the High Frequency Electronic Lab (HFE-Lab) at the University of Perugia. 